\title{
One-dimensional bargaining with a general voting rule
}

Citation for published version (APA):

Predtetchinski, A. (2007). One-dimensional bargaining with a general voting rule. METEOR, Maastricht University School of Business and Economics. METEOR Research Memorandum No. 045 https://doi.org/10.26481/umamet.2007045

Document status and date:

Published: 01/01/2007

DOI:

10.26481/umamet.2007045

Document Version:

Publisher's PDF, also known as Version of record

\section{Please check the document version of this publication:}

- A submitted manuscript is the version of the article upon submission and before peer-review. There can be important differences between the submitted version and the official published version of record.

People interested in the research are advised to contact the author for the final version of the publication, or visit the DOI to the publisher's website.

- The final author version and the galley proof are versions of the publication after peer review.

- The final published version features the final layout of the paper including the volume, issue and page numbers.

Link to publication

\footnotetext{
General rights rights.

- You may freely distribute the URL identifying the publication in the public portal. please follow below link for the End User Agreement:

www.umlib.nl/taverne-license

Take down policy

If you believe that this document breaches copyright please contact us at:

repository@maastrichtuniversity.nl

providing details and we will investigate your claim.
}

Copyright and moral rights for the publications made accessible in the public portal are retained by the authors and/or other copyright owners and it is a condition of accessing publications that users recognise and abide by the legal requirements associated with these

- Users may download and print one copy of any publication from the public portal for the purpose of private study or research.

- You may not further distribute the material or use it for any profit-making activity or commercial gain

If the publication is distributed under the terms of Article $25 \mathrm{fa}$ of the Dutch Copyright Act, indicated by the "Taverne" license above, 
Arkadi Predtetchinski

One-dimensional bargaining with a general voting rule

$\mathrm{RM} / 07 / 045$

JEL code: C78

\section{METE@R}

Maastricht research school of Economics of TEchnology and ORganizations

Universiteit Maastricht

Faculty of Economics and Business Administration P.O. Box 616

NL - 6200 MD Maastricht

phone : ++31433883830

fax : :+31433884873 


\title{
One-dimensional bargaining with a general voting rule*
}

\author{
Arkadi Predtetchinski ${ }^{\dagger}$
}

October 22, 2007

\begin{abstract}
We study a model of multilateral bargaining over social outcomes represented by points in the unit interval. An acceptance or rejection of a proposal is determined by a voting rule as represented by a collection of decisive coalitions. The focus of the paper is on the asymptotic behavior of subgame perfect equilibria in stationary strategies as the discount factor goes to one. We show that, along any sequence of stationary subgame perfect equilibria, as the discount factor goes to one, the social acceptance set collapses to a point. This point, called the bargaining outcome, is independent of the sequence of equilibria and is uniquely determined by the set of players, the utility functions, the recognition probabilities, and the voting rule. The central result of the paper is a characterization of the bargaining outcome as a unique zero of the characteristic equation.
\end{abstract}

JEL classification code: $\mathrm{C} 78$

Keywords: Bargaining, subgame perfect equilibrium, voting rules.

${ }^{*}$ The author is most thankful to Jean-Jacques Herings and the participants of the Mathematical Economics Seminar in the Sobolev Institute of Mathematics (Novosibirsk, Russia).

${ }^{\dagger}$ Department of Economics, Maastricht University, P.O. Box 616, 6200 MD Maastricht, The Netherlands. E-mail: A.Predtetchinski@algec.unimaas.nl 


\section{Introduction}

This paper analyzes a model of multilateral bargaining where players must choose one alternative from a set of alternatives represented by points in the unit interval. An alternative might be a level of taxation, a location of a facility, or an index of an ideological content of a policy (left vs. right).

Bargaining proceeds as follows. At the beginning of each period, nature randomly selects one of the players as a proposer. The probability for a player to become a proposer, the so-called recognition probability, is assumed to be the same in each period. The player chosen by nature puts forward a proposal that specifies one alternative. All players then react to the proposal. Each player can either reject or accept the proposal. The votes are cast sequentially, the sequence of responses being fixed throughout the game. Whether the proposal passes or fails is then determined by a voting rule, as represented by a collection of decisive coalitions. The passing of a proposal requires an approval of it by all the players in some decisive coalition. Examples of voting rules include the unanimity acceptance rule when a passing of a proposal requires an approval of it by all the players, the quota rule, when a fixed number of votes is needed for a passing of a proposal, or the simple majority rule. If the proposal passes, it is implemented and the game ends. In this case each player receives a discounted utility of the alternative. Otherwise, a new period begins.

We consider subgame perfect equilibria in stationary strategies. Stationarity means that a proposal of any player does not depend on the history of play and a player's reaction to a proposal depends only on the proposal itself. The focus of the paper is on the asymptotic behavior of stationary subgame perfect equilibria as the discount factor approaches one.

The results are as follows. We prove that, along any sequence of subgame perfect equilibria in stationary strategies the social acceptance set collapses to a point. This point, called the bargaining outcome, is independent of the sequence of equilibria and is uniquely determined by the set of players, the utility functions, the recognition probabilities, and the voting rule. The central result of the paper is a characterization of the bargaining outcome as a unique zero of the characteristic equation. This paper is the first to provide the characterization of the limit of stationary equilibrium in a one-dimensional model of bargaining.

The results are obtained under rather minimal assumptions. Thus the instantaneous utility functions are only assumed to be single-peaked and concave. Furthermore, we require that the intersection of any two decisive coalitions contain a player with a positive recognition probability. This requirement puts but a very mild restriction on the voting rule and the recognition probabilities.

Games of bargaining with a one-dimensional space of alternatives have been previously considered in Banks and Duggan [1], Cho and Duggan [4], Kalandrakis [7], and Cardona and Ponsati [3]. Banks and Duggan [1] and Kalandrakis [7] consider bargaining in a situation where the alternatives are represented by points in a general compact convex set. For the special case where the set of alternatives is one-dimensional Banks and Duggan [1] establish the existence of stationary subgame perfect equilibria in pure strategies, while Kalandrakis [7] shows that pure strategy stationary subgame perfect equilibria are locally 
unique and finite in number for almost all discount factors. Cho and Duggan [4] prove uniqueness of stationary subgame perfect equilibria in pure strategies in a model of onedimensional bargaining under the assumption that the utility functions are quadratic. They also establish that any pair of stationary subgame perfect equilibria are nested in the sense that the social acceptance set in one equilibrium forms the subset of the social acceptance set of the other equilibrium.

The game tree used in this paper is the same as in Banks and Duggan [1], Cho and Duggan [4] and Kalandrakis [7] except that the players vote sequentially, not simultaneously. This difference turns out to be inessential, and the results of Banks and Duggan [1] and Cho and Duggan [4] are true in the setting of the present paper. The results of Kalandrakis [7] on local uniqueness and finiteness equilibria, on the other hand, are generic in the discount factors that may be different across the players. We restrict our attention to the case of a common discount factor.

Cardona and Ponsati [3] also study bargaining over a one-dimensional set of alternatives but their model differs from ours in two respects. Firstly, in Cardona and Ponsati [3] the identity of the proposer rotates deterministically in the player set, whereas we use timeindependent recognition probabilities. And, secondly, Cardona and Ponsati [3] restrict attention to a quota voting rule, whereas we use a more general family of voting rules. Cardona and Ponsati [3] prove, in their setting, the asymptotic uniqueness of subgame perfect equilibria in stationary strategies, but do not provide the characterization.

Our paper complements these insights by providing the asymptotic uniqueness result, and more importantly, the explicit computation of the limit of subgame perfect equilibria in stationary strategies.

There are numerous other contributions that study stationary subgame perfect equilibria in various games of multilateral bargaining under the assumption that the the boundary of the set of feasible payoffs is of dimension $n-1$, where $n$ is the number of players. This assumption is satisfied in a situation when $n$ players have to divide an amount of money amongst themselves, in which case the set of alternatives consists of all feasible redistributions of an amount among $n$ players. Thus Merlo and Wilson [9] and Merlo and Wilson [10] give sufficient conditions for the uniqueness of stationary subgame perfect equilibria (in pure strategies) in a model where the identity of the proposal and the amount of money to be divided follow a Markov process and the unanimous approval is needed for a proposal to pass. Eraslan [5] establishes uniqueness of subgame perfect equilibria (in mixed strategies) in a game with a tree similar to the one described above and a quota voting rule. Eraslan and Merlo [6] characterize stationary subgame perfect equilibria (in mixed strategies) in a model where the amount of money to be divided is stochastic and a general agreement rule is used. It is clear that the game where the payoffs are generated by a one-dimensional space of alternatives is not covered by this analysis.

The idea of studying the asymptotic behavior of subgame perfect equilibria in the bargaining context goes back to Binmore et al [2] who prove that in a two-player game, the limit of subgame perfect equilibrium is given by the Nash bargaining solution. Miyakawa [11] generalizes the result of Binmore et al [2]. He obtains a characterization of the limit of stationary subgame perfect equilibria in the $n$-player game with the unanimity acceptance 
rule as the probability of the breakdown of the negotiations goes to zero, under the assumption that the boundary of the set of feasible utility vectors is an $(n-1)$-dimensional surface. Miyakawa [11] shows that the limit is the asymmetric Nash bargaining solution, with the weights corresponding to the recognition probabilities. A related result is given in Kultti and Vartiainen [8], who also assume that the boundary of the feasible payoffs is $(n-1)$-dimensional surface. They obtain the (asymmetric) Nash bargaining solution as a limit of the Von Neumann-Morgenstern stable set as the discount factor vanishes. The stable set is defined with respect to a dominance relation where an alternative $x$ dominates an alternative $y$ if some player prefers $x$ even with a one-period delay. Our paper provides the asymptotic results for the stationary subgame perfect equilibrium in a setting that has not been covered by the analysis in Binmore et al [2], Miyakawa [11] or Kultti and Vartiainen [8].

The outline of the paper is as follows. Section 2 introduces the game of bargaining and the concept of $\delta$-equilibrium. Essentially, $\delta$-equilibrium is a subgame perfect equilibrium in stationary strategies where each player is assumed to vote sincerely. Section 3 examines a relationship of the $\delta$-equilibrium and the subgame perfect equilibrium. It is shown that each $\delta$-equilibrium is a subgame perfect equilibrium in stationary strategies. Conversely, given a subgame perfect equilibrium in stationary strategies there exists a $\delta$-equilibrium having the same equilibrium proposals and inducing the same equilibrium payoffs. In this sense, the set of $\delta$-equilibria is effectively the same as the set of subgame perfect equilibria in stationary strategies.

In Section 4 we establish that, along any sequence of $\delta$-equilibria, as the discount factor converges to one, the social acceptance set collapses to a point. Any such point is called a bargaining outcome. Section 5 introduces the characteristic function and the main result of the paper: there is a unique bargaining outcome being the (unique) generalized zero of the characteristic function. Section 6 provides the proof of the main result.

\section{The model}

We study a world $\omega$ described by the following variables: $X, N, \mu, \mathscr{C}$ and $u_{\bullet}$. The symbol $X$ denotes the unit interval $[0,1]$. This is a space of alternatives or social states the players must choose from. The set $N$ is a finite set of players and $\mu$ is a probability distribution on $N$. The probability $\mu(i)$ is a recognition probability of player $i$, that is the probability for player $i$ to be chosen a proposer. The symbol $u_{\bullet}$ denotes a collection of utility functions, one for each player. The utility function of player $t \in N$ is $u_{t}: X \rightarrow[0,1]$. We shall assume that the utility functions satisfy the following assumption.

(A1) For each $t \in N$ the utility function $u_{t}: X \rightarrow[0,1]$ is concave, continuous, and it attains its unique maximum at point $\bar{x}_{t}$.

The symbol $\mathscr{C}$ is a collection of subsets of $N$ representing a voting rule. The sets in $\mathscr{C}$ are decisive coalitions in the sense that an approval of a proposal by any of these coalitions is sufficient for a passing of a proposal. For instance, if the passing of a proposal requires 
the approval of it by each player, then the collection $\mathscr{C}$ consists of the set $N$ alone. If the acceptance of a proposal requires the approval of it by at least $q$ voters, then the the collection $\mathscr{C}$ consists of all sets $C \subset N$ such that $|C|=q$. In particular, the simple majority rule is a quota rule where the quota $q$ equals $n+1$ in a game with $2 n+1$ players. An interesting voting rule is an adaptation of the rule used by the United Nations Security Council. The Council consists of five permanent and ten temporary members, and the passing of its resolutions requires the yes-votes of at least nine members. Furthermore, each permanent member has a veto right, which means that the no-vote by any of the permanent members overrules any majority. Letting $P$ denote the set of permanent members, the collection $\mathscr{C}$ consists of all sets $C \subset N$ such that $P \subset C$ and $|C|=9$. We shall assume the following.

(A2) For each $C_{1}$ and $C_{2}$ in $\mathscr{C}$ the inequality $\mu\left(C_{1} \cap C_{2}\right)>0$ holds.

A restriction on the collection $\mathscr{C}$ of decisive coalitions commonly employed in the literature on voting is that for each $C_{1}$ and $C_{2}$ in $\mathscr{C}$ the set $C_{1} \cap C_{2}$ be non-empty. Assumption (A2) not only requires that the set $C_{1} \cap C_{2}$ be non-empty, but also that it contain a player having a positive probability of recognition. Assumption (A2) is trivially satisfied in the case of the unanimity acceptance rule, i.e. when $\mathscr{C}=\{N\}$. If $|N|$ is an odd number and the collection $\mathscr{C}$ represents the simple majority rule, then assumption (A2) implies that all players's recognition probabilities are positive, because for each $i \in N$ there exist two decisive coalitions $C_{1}$ and $C_{2}$ such that $C_{1} \cap C_{2}=\{i\}$. If $\mathscr{C}$ represents the voting rule of the Security Council as described above, for the assumption (A2) to be satisfied it is sufficient that at least one permanent member have a positive recognition probability.

Given a discount factor $\delta \in[0,1]$ we define a game of bargaining $\Gamma(\delta)$ as follows. The game starts in period zero. Each period $\tau$ begins with nature randomly choosing a player from the set $N$ to make a proposal. Player $i$ is chosen by nature with probability $\mu(i)$. The chosen player proposes an alternative $x$ from $X$. All players (including the proposer) respond sequentially, according to a total order $>$ on the player set $N$. The order $>$ is fixed throughout the game. Each responder can either accept or reject the current proposal. If all players in some decisive coalition $C \in \mathscr{C}$ accept the proposal, the game terminates and the proposal is implemented. Otherwise, period $\tau+1$ begins.

If alternative $x$ is agreed upon in period $\tau$, player $i$ receives a payoff of $\delta^{\tau} u_{i}(x)$. The payoff of perpetual disagreement to any player is zero.

The game tree of $\Gamma(\delta)$ is similar to that in Banks and Duggan [1] with the difference that in the game $\Gamma(\delta)$ the players vote sequentially, while in [1] the votes are cast simultaneously. We insist that the players vote sequentially to rule out coordination problems in the response stage of the game, for example a situation when any point in the set $X$ is unanimously accepted independently of preferences.

We shall restrict our attention to stationary strategies by which we mean the strategies such that (a) a proposal of any player is independent of the history of play and (b) the reaction of a player to a proposal only depends on the proposal itself. Thus a stationary strategy of player $t$ consists of a proposal $x_{t}$ and an acceptance set $A_{t}$. Player $t$ proposes the point $x_{t}$ whenever player $t$ is chosen to be a proposer and he accepts a proposal $x$ if 
and only if $x$ is an element of the set $A_{t}$. A joint stationary strategy $\sigma=\left(x_{\bullet}, A_{\bullet}\right)$ induces the acceptance set $A_{C}=\cap_{t \in C} A_{t}$ of coalition $C$ and the social acceptance set $A=\cup_{C \in \mathscr{C}} A_{C}$. A proposal $x$ is implemented if and only if it is an element of the social acceptance set $A$.

Consider a case where the proposal $x_{i}$ of each player $i$ lies in the social acceptance set $A$. In this case each proposal is accepted without delay. The expected utility $y_{t}$ of player $t$ can then be computed as a weighted sum of $u_{t}\left(x_{i}\right)$, where the weights are equal to the respective recognition probabilities. The utility $y_{t}$ is also player $t$ 's continuation payoff when a proposal is turned down. Therefore, player $t$ accepts a proposal $x$ if and only if $u_{t}(x) \geq \delta y_{t}$. Since all proposals in the set $A$ pass, player $t$ proposes a point $x_{t}$ that maximizes player $t$ 's utility on $A$. These considerations motivate the following definition.

Definition 1 A joint stationary strategy $\sigma=\left(x_{\bullet}, A_{\bullet}\right)$ is a $\delta$-equilibrium of the world $\omega$ if there exist the numbers $\left(y_{t}\right)_{t \in N}$, the sets $\left(A_{C}\right)_{C \in \mathscr{C}}$ and the set $A$ such that the following conditions are satisfied:

$$
\begin{gathered}
x_{t}=\arg \max _{x \in A} u_{t}(x) \text { for each } t \in N, \\
y_{t}=\sum_{i \in N} \mu(i) u_{t}\left(x_{i}\right) \text { for each } t \in N, \\
A_{t}=\left\{x \in X \mid u_{t}(x) \geq \delta y_{t}\right\} \text { for each } t \in N, \\
A_{C}=\cap_{t \in C} A_{t} \text { for each } C \in \mathscr{C}, \\
A=\cup_{C \in \mathscr{C}} A_{C} .
\end{gathered}
$$

A $\delta$-equilibrium can be seen to be equivalent to the definition of stationary equilibrium (in pure strategies) in Banks and Duggan [1] and Cho and Duggan [4]. In particular, the existence of $\delta$-equilibrium is established in Theorem 2 in Banks and Duggan [1]. Cho and Duggan [4] show that $\delta$-equilibrium is unique for each $\delta<1$ under the assumption that the utility functions $u_{i}$ are quadratic and the voting rule $\mathscr{C}$ is proper and strong. In general, there may be multiple $\delta$-equilibria, an example being given in Cho and Duggan [?].

Since we assume the utility functions to be concave, each individual acceptance set is a closed interval. We shall use the notation $\left[x_{t}^{-}, x_{t}^{+}\right]$to denote the individual acceptance set $A_{t}$ of player $t$. The acceptance set of coalition $C$ is also a closed interval, denoted by $\left[x_{C}^{-}, x_{C}^{+}\right]$. Furthermore,

$$
x_{C}^{-}=\max _{t \in C}\left\{x_{t}^{-}\right\} \text {and } x_{C}^{+}=\min _{t \in C}\left\{x_{t}^{+}\right\} .
$$

We write $E\left(x_{\bullet}\right)$ to denote the expected proposal, that is

$$
E\left(x_{\bullet}\right)=\sum_{i \in N} \mu(i) x_{i}
$$

Proposition 1 Let $\left(x_{\bullet}, A_{\bullet}\right)$ be a $\delta$-equilibrium inducing the social acceptance set $A$. Then $A$ is a closed interval containing the point $E\left(x_{\bullet}\right)$. 


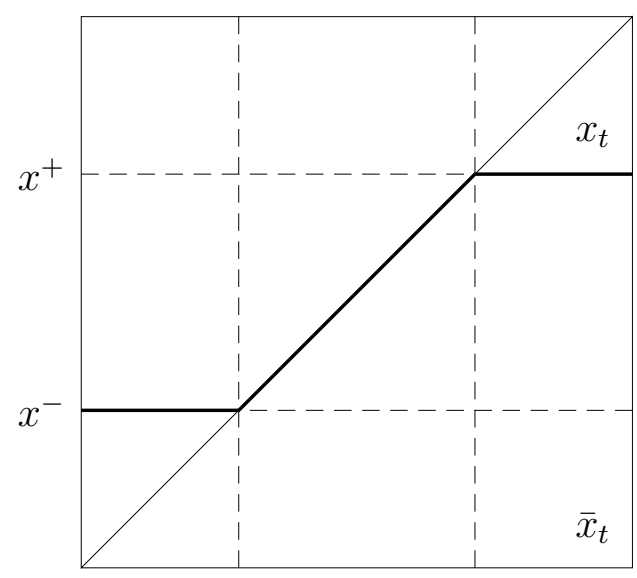

Figure 1: Equilibrium proposals.

Proof. Each individual acceptance set $A_{t}$ contains the point $E\left(x_{\bullet}\right)$, because

$$
u_{t}\left(E\left(x_{\bullet}\right)\right) \geq E\left(u_{t}\left(x_{\bullet}\right)\right)=y_{t} \geq \delta y_{t}
$$

by the concavity of the function $u_{t}$. It follows that the acceptance set $A_{C}$ of each coalition $C$ contains the point $E\left(x_{\bullet}\right)$. A finite union of closed intervals having a point in common is obviously closed interval.

We let the social acceptance set be $\left[x_{-}, x_{+}\right]$, where the endpoints can be computed as

$$
x_{-}=\min _{C \in \mathscr{C}}\left\{x_{C}^{-}\right\} \text {and } x_{+}=\max _{C \in \mathscr{C}}\left\{x_{C}^{+}\right\} .
$$

The equilibrium proposal $x_{t}$ of player $t$ is a point of $\left[x_{-}, x_{+}\right]$closest to $\bar{x}_{t}$, the ideal point of individual $t$. Figure 1 illustrates. Thus

$$
x_{t}= \begin{cases}x_{-} & \text {if } \bar{x}_{t} \leq x_{-} \\ \bar{x}_{t} & \text { if } x_{-} \leq \bar{x}_{t} \leq x_{+} \\ x_{+} & \text {if } x_{+} \leq \bar{x}_{t}\end{cases}
$$

Definition 2 For each natural $n$ let $A^{n}$ be a social acceptance set in a $\delta^{n}$-equilibrium of the world $\omega$ and let $x^{n}$ be a point of $A^{n}$. Suppose that the sequence $\delta^{n}$ converges to one and $x^{n}$ converges to a point $x$. Then the alternative $x$ is called a bargaining outcome of $\omega$. A collection of bargaining outcomes is called a bargaining solution.

The main result of the paper states that the bargaining outcome is unique. In particular, along any sequence of $\delta$-equilibria, as $\delta$ converges to one, the social acceptance set collapses to a point, and in the limit all players make the same proposal. 


\section{Subgame perfect equilibria in stationary strategies}

In this section we show that each $\delta$-equilibrium is a subgame perfect equilibrium of the game $\Gamma(\delta)$. Conversely, given a subgame perfect equilibrium in stationary strategies there exists a $\delta$-equilibrium having the same equilibrium proposals and inducing the same expected payoffs. In particular, each subgame perfect equilibrium in stationary strategies is a no-delay equilibrium. In this sense, the set of $\delta$-equilibria effectively represents all subgame perfect equilibria in stationary strategies. One can say that $\delta$-equilibrium is a subgame perfect equilibrium in stationary strategies where each player votes sincerely.

Theorem 1 Each $\delta$-equilibrium is a subgame perfect equilibrium of the game $\Gamma(\delta)$.

The proof of Theorem 1 consists of two steps. The first step is to show that the strategy $\sigma$ is robust to all one-shot deviations. Recall that a strategy $\bar{\sigma}_{t}$ for player $t$ is said to be a one-shot deviation from $\sigma$ at node $h$ if it coincides with the strategy $\sigma_{t}$ on all nodes but $h$. The second step establishes the one-shot deviation property for the game $\Gamma(\delta)$ : if there is a profitable deviation from the joint strategy $\sigma$, then there is a profitable one-shot deviation from $\sigma$.

Proposition 2 Let $\sigma=\left(x_{\bullet}, A_{\bullet}\right)$ be a $\delta$-equilibrium. Then no player has a one-shot profitable deviation from $\sigma$ at any node of the game $\Gamma$.

Proof. Let $\bar{\sigma}_{t}$ be a one-shot deviation from $\sigma_{t}$ at node $h$. Let $\Gamma(h)$ be the subgame that starts at node $h$.

Suppose player $t$ has to make a proposal at node $h$. Under the strategy $\sigma_{t}$ player $t$ proposes the alternative $x_{t}$, which is accepted, leading to a payoff of $u_{t}\left(x_{t}\right)$ for player $t$. Suppose under the strategy $\bar{\sigma}_{t}$ player $t$ makes a proposal $x$. If $x$ is not an element of the social acceptance set $A=\cup_{C \in \mathscr{C}} \cap_{i \in C} A_{i}$, the proposal $x$ will be rejected. As $\bar{\sigma}_{t}$ coincides with $\sigma_{t}$ on all nodes following $h$, player $t$ will receive a payoff of $\delta y_{t}$. By the definition of $\delta$-equilibrium, $\delta y_{t} \leq u_{t}\left(x_{t}\right)$. If $x$ is an element of $A$, then the proposal $x$ is accepted and player $t$ receives a payoff of $u_{t}(x)$. However, $u_{t}(x) \leq u_{t}\left(x_{t}\right)$, because by the definition of $\delta$-equilibrium, $x_{t}$ maximizes the function $u_{t}$ on the set $A$. We conclude that the strategy $\bar{\sigma}_{t}$ does not give a higher payoff than the strategy $\sigma_{t}$ in the subgame $\Gamma(h)$.

Suppose player $t$ has to react to a proposal $x$ at node $h$. Suppose that $u_{t}(x) \geq \delta y_{t}$. Then $x \in A_{t}$, and the strategy $\sigma_{t}$ accepts the proposal $x$. The strategy $\bar{\sigma}_{t}$ rejects the proposal $x$, because it is assumed to be a deviation from $\sigma_{t}$ at the node $h$. If in the subgame $\Gamma(h)$ the joint strategy $\left(\sigma_{t}, \sigma_{-t}\right)$ leads to a social rejection of the proposal $x$, so does the joint strategy $\left(\bar{\sigma}_{t}, \sigma_{-t}\right)$, since the latter gives one less yes-vote to $x$ than the former. In that case the payoff to player $t$ in the subgame $\Gamma(h)$ is $\delta y_{t}$, whether player $t$ uses the strategy $\sigma_{t}$ or $\bar{\sigma}_{t}$. Suppose that in the subgame $\Gamma(h)$ the joint strategy $\left(\sigma_{t}, \sigma_{-t}\right)$ leads to a social acceptance of the proposal $x$, so the payoff to player $t$ on the strategy $\sigma_{t}$ is $u_{t}(x)$. The payoff on the strategy $\bar{\sigma}_{t}$ in the subgame $\Gamma(h)$ is either $u_{t}(x)$ or $\delta y_{t}$, depending on whether the joint strategy $\left(\bar{\sigma}_{t}, \sigma_{-t}\right)$ leads to a social acceptance or rejection of the proposal $x$, but in either case is not higher than the payoff on the strategy $\sigma_{t}$. 
Suppose that $u_{t}(x)<\delta y_{t}$. Then $x$ is not in $A_{t}$, so the strategy $\sigma_{t}$ rejects the proposal $x$, and the strategy $\bar{\sigma}_{t}$ accepts the proposal $x$ at the node $h$. If in the subgame $\Gamma(h)$ the joint strategy $\left(\sigma_{t}, \sigma_{-t}\right)$ leads to a social acceptance of the proposal $x$, so does the joint strategy $\left(\bar{\sigma}_{t}, \sigma_{-t}\right)$, since under the joint strategy $\left(\bar{\sigma}_{t}, \sigma_{-t}\right)$ the proposal $x$ receives one more yes-vote than under the strategy $\left(\sigma_{t}, \sigma_{-t}\right)$. In that case the payoff to player $t$ in the subgame $\Gamma(h)$ is $u_{t}(x)$ on both strategies $\sigma_{t}$ and $\bar{\sigma}_{t}$. Suppose that in the subgame $\Gamma(h)$ the joint strategy $\left(\sigma_{t}, \sigma_{-t}\right)$ leads to a social rejection of the proposal $x$, so the payoff to player $t$ on the strategy $\sigma_{t}$ is $\delta y_{t}$. The payoff on the strategy $\bar{\sigma}_{t}$ in the subgame $\Gamma(h)$ is either $u_{t}(x)$ or $\delta y_{t}$, depending on whether the joint strategy $\left(\bar{\sigma}_{t}, \sigma_{-t}\right)$ leads to a social acceptance or rejection of the proposal $x$. Therefore $\bar{\sigma}_{t}$ does not give a higher payoff than the strategy $\sigma_{t}$ in the subgame $\Gamma(h)$.

For the proof of Proposition 3 the reader can refer to Predtetchinski [12, Proposition 2].

Proposition 3 Let $\sigma$ be a profile of strategies. If player thas a profitable deviation from $\sigma$, then player $t$ has a profitable one-shot deviation from $\sigma$.

We now compute the payoffs induced by a joint stationary strategy $\sigma=\left(x_{\bullet}, A_{\bullet}\right)$. Let $y_{t}$ denote the expected payoff to player $t$ at the beginning of the game induced by $\sigma$. Let $N_{a}=\left\{t \in N \mid x_{t} \in A\right\}$ be the set of players whose proposal is accepted under $\sigma$ and $N_{r}=\left\{t \in N \mid x_{t} \notin A\right\}$ denote the set of players whose proposal is rejected. If nature chooses a proposer $i$ from $N_{a}$, player $t$ receives a payoff of $u_{t}\left(x_{i}\right)$, while if nature chooses a proposer from $N_{r}$, then player $t$ 's payoff is $\delta y_{t}$. Thus $y_{t}$ satisfies the following relation:

$$
y_{t}=\sum_{i \in N_{a}} \mu(i) u_{t}\left(x_{i}\right)+\delta \mu\left(N_{r}\right) y_{t}
$$

Solving the equation, we obtain the following expression for $y_{t}$ :

$$
y_{t}= \begin{cases}\frac{1}{1-\delta \mu\left(N_{r}\right)} \sum_{i \in N_{a}} \mu(i) u_{t}\left(x_{i}\right) & \text { if } \mu\left(N_{a}\right)>0 \\ 0 & \text { otherwise. }\end{cases}
$$

We now turn to the converse of Theorem 1. It is clear that there always exist subgame perfect equilibria in stationary strategies that are not $\delta$-equilibria. To illustrate, take a $\delta$-equilibrium $\sigma$ and suppose that the alternative $x$ is not in the social acceptance set induced by $\sigma$. Let $T$ be the set of players who reject the alternative $x$. Let $i$ be a player who reacts to a proposal before any player in the set $T$. Then, at any node of the game $\Gamma$ where player $i$ has to respond to a proposal $x$, player $i$ is indifferent between accepting the proposal $x$ and rejecting it, since it will later be rejected by the players in the set $T$. Consequently, we can remove the alternative $x$ from the individual acceptance set of player $i$. The resulting profile of strategies will still be a subgame perfect equilibrium, but not a $\delta$-equilibrium. 


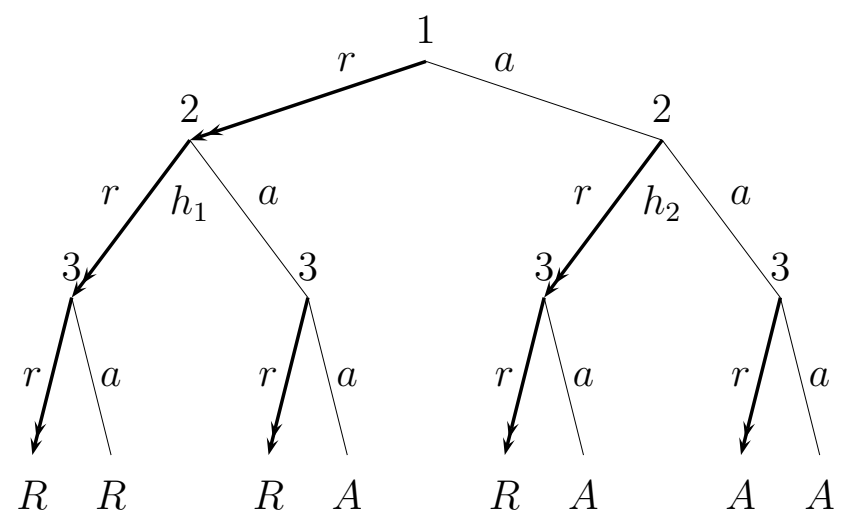

Figure 2: The response stage of the game $\Gamma$.

Thus we will not be able to show that each subgame perfect equilibrium in stationary strategies is a $\delta$-equilibrium. However, we shall prove that, given a subgame perfect equilibrium in stationary strategies, there exists a $\delta$-equilibrium inducing the same expected payoffs, having the same equilibrium proposals, and (provided that there are players whose ideal points are zero and one) the same social acceptance set.

Important for the proof of Theorem 2 is the concept of pivotal vote. Suppose one is given a joint stationary strategy $\sigma$. Consider a node $h$ of the game $\Gamma$ where player $t$ has to react to some proposal, say $x$. Player $t$ 's vote is said to be pivotal at node $h$ if it determines the social acceptance or rejection of the proposal $x$.

We illustrate the idea of pivotal vote by means of an example. Suppose there are three players, and the yes-votes of any two players are sufficient for a passing of a proposal. The order of response is $1<2<3$. Suppose that under the given joint stationary strategy the proposal $x$ is rejected by all three players. Figure 2 depicts the response stage of the game $\Gamma$. The capital letters in Figure 2 indicate whether the proposal in question is socially accepted or not, and the arrows indicate the strategies.

The player 2's vote at the node $h_{2}$ is pivotal. Indeed, if player 2 accepts at the node $h_{2}$, then the proposal $x$ will be socially accepted, and if player 2 rejects, the proposal will be turned down. Notice that player 2's vote at node $h_{1}$ is not pivotal, because the proposal will be socially rejected, whether player 2 votes for or against it. In the same way, player 1 is not pivotal at his decision node.

Definition 3 Let $\sigma$ be a joint stationary strategy. Consider a node $h$ of the game $\Gamma$ where player $t$ has to react to some proposal, say $x$. Let the $\sigma_{t}^{\prime}$ and $\sigma_{t}^{\prime \prime}$ be strategies for player $t$ that coincide with the strategy $\sigma_{t}$ at any node of the game $\Gamma$ but $h$, where $\sigma_{t}^{\prime}$ accepts $x$ and $\sigma_{t}^{\prime \prime}$ rejects $x$. Player $t$ is said to be pivotal at node $h$ if the proposal $x$ is socially accepted on the path of play induced by the joint strategy $\left(\sigma_{t}^{\prime}, \sigma_{-t}\right)$ in the subgame $\Gamma(h)$ and is socially rejected on the path of play induced by the joint strategy $\left(\sigma_{t}^{\prime \prime}, \sigma_{-t}\right)$ in the subgame $\Gamma(h)$. 
If all players were voting sincerely, we would expect player $t$ to accept a proposal $x$ if the utility of $x$ to player $t$ is higher than $t$ 's continuation utility and to reject it if the utility of $x$ is lower than the continuation utility. In fact, the players need not vote sincerely, even in a subgame perfect equilibrium. Consider again Figure 2. Since player 1's vote is irrelevant, both rejecting and accepting the proposal are player 1's best responses to player 2's and 3's strategies. Thus player 1 may well reject a proposal $x$ even even though the utility of $x$ is higher than player 1's continuation utility. On the other hand, player 2's vote is decisive at $h_{2}$, so player 2 receives a utility of $\delta y_{2}$ if he chooses to reject $x$ and a utility of $u_{2}(x)$ if he chooses to accept it. Since in a subgame perfect equilibrium player 2 should maximize his utility at any node of the game, including the node $h_{2}$, player 2 should accept the proposal $x$ if $u_{2}(x)>\delta y_{2}$ and reject it if $u_{2}(x)<\delta y_{2}$.

It is therefore important to know whether a player is ever pivotal or not. This is answered by Proposition 4 below.

Proposition 4 Let $\sigma=\left(x_{\bullet}, A_{\bullet}\right)$ be a joint stationary strategy in the game $\Gamma$ inducing the acceptance set $A_{C}$ for coalition $C$ and the social acceptance set $A$. Let $x \in X$.

(a) Suppose $x$ is not an element of $A$. Then for each $C \in \mathscr{C}$ there is a player $t \in C$ such that $x$ is not an element of the set $A_{t}$ and player $t$ 's vote is pivotal at some node $h$ of the game $\Gamma$.

(b) Suppose $x$ is an element of $A$. Then there exists a $C \in \mathscr{C}$ such that $x$ is an element of $A_{C}$ and for each $t \in C$ player $t$ 's vote is pivotal at some node $h$ of the game $\Gamma$.

The proof of Proposition 4 is very involved and is relegated to the appendix. We are now in a position to prove Theorem 2 .

Theorem 2 Suppose $0 \leq \delta<1$. Consider a joint strategy $\sigma=\left(x_{\bullet}, A_{\bullet}\right)$ inducing the expected payoffs $y_{\bullet}$ and the social acceptance set $A$. Suppose $\sigma$ is a subgame perfect equilibrium of the game $\Gamma(\delta)$. Then there exists a $\delta$-equilibrium $\left(x_{\bullet}, A_{\bullet}^{\prime}\right)$ inducing the expected payoffs $y_{\bullet}$ and the social acceptance set $A^{\prime}$ such that $\operatorname{Int} A^{\prime} \subset A \subset A^{\prime}$. Furthermore, if there are players $i^{0}$ and $i^{1}$ such that $\bar{x}_{i^{0}}=0$ and $\bar{x}_{i^{1}}=1$, then $A=A^{\prime}$.

Proof. Define the following sets:

$$
\begin{aligned}
& A_{i}^{\prime}=\left\{x \in X \mid u_{i}(x) \geq \delta y_{i}\right\} \quad A_{i}^{\prime \prime}=\left\{x \in X \mid u_{i}(x)>\delta y_{i}\right\} \\
& A_{C}^{\prime}=\cap_{i \in C} A_{i}^{\prime} \quad A_{C}^{\prime \prime}=\cap_{i \in C} A_{i}^{\prime \prime} \\
& A^{\prime}=\cup_{C \in \mathscr{C}} A_{C}^{\prime} \quad A^{\prime \prime}=\cup_{C \in \mathscr{C}} A_{C}^{\prime \prime} \text {. }
\end{aligned}
$$

Step 1. We prove the inclusion $A^{\prime \prime} \subset A$.

Suppose $x$ is not an element of $A$. Take a $C \in \mathscr{C}$. By part (a) of Proposition 4, there is a player $t \in C$ such that $x$ is not in $A_{t}$ and player $t$ 's vote is pivotal at a node $h$ of the game $\Gamma$. We now prove that $x$ is not an element of the set $A_{t}^{\prime \prime}$. It then follows that it is not an element of the set $A_{C}^{\prime \prime}$, and, since $C$ is an an arbitrary coalition in $\mathscr{C}$, that it is not an element of the set $A^{\prime \prime}$. 
Let $\sigma_{t}^{\prime}$ and $\sigma_{t}^{\prime \prime}$ be as in Definition 3. Thus $\sigma_{t}^{\prime}$ and $\sigma_{t}^{\prime \prime}$ coincide with the strategy $\sigma_{t}$ at any node of the game $\Gamma$ but $h$, where $\sigma_{t}^{\prime}$ accepts $x$ and $\sigma_{t}^{\prime \prime}$ rejects $x$. Then the proposal $x$ is socially accepted on the path of play of the joint strategy $\left(\sigma_{t}^{\prime}, \sigma_{-t}\right)$ in the subgame $\Gamma(h)$ and is rejected on the path of play of the joint strategy $\left(\sigma_{t}^{\prime \prime}, \sigma_{-t}\right)$. It follows that the payoff to player $t$ on his strategy $\sigma_{t}^{\prime}$ in the subgame $\Gamma(h)$ is $u_{t}(x)$ and the payoff on the strategy $\sigma_{t}^{\prime \prime}$ is $\delta y_{t}$. Now since $x$ is not an element of $A_{t}$, player $t$ rejects the proposal $x$ under the strategy $\sigma_{t}$, so in fact $\sigma_{t}=\sigma_{t}^{\prime \prime}$. Since $\sigma$ induces a Nash equilibrium in the subgame $\Gamma(h)$, we must have that $u_{t}(x) \leq \delta y_{t}$. Thus $x$ is not an element of the set $A_{t}^{\prime \prime}$, as desired.

Step 2. We prove the inclusion $A \subset A^{\prime}$.

Let $x$ be an element of $A$. By part (b) of Proposition 4, there exists a $C \in \mathscr{C}$ such that $x$ is an element of $A_{C}$ and for each $t \in C$ player $t$ 's vote is pivotal at some node $h$ of the game $\Gamma$. We now prove that $x$ is an element of $A_{C}^{\prime}$.

As before, let $\sigma_{t}^{\prime}$ and $\sigma_{t}^{\prime \prime}$ be as in Definition 3. Then the payoff to player $t$ on his strategy $\sigma_{t}^{\prime}$ in the subgame $\Gamma(h)$ is $u_{t}(x)$ and the payoff on the strategy $\sigma_{t}^{\prime \prime}$ is $\delta y_{t}$. Since $x$ is an element of $A_{t}$, player $t$ accepts the proposal $x$ under the strategy $\sigma_{t}$, so in fact $\sigma_{t}=\sigma_{t}^{\prime}$. Since $\sigma$ induces a Nash equilibrium in the subgame $\Gamma(h)$, we must have that $\delta y_{t} \leq u_{t}(x)$. It follows that $x \in A_{t}^{\prime}$. Since $t$ is an arbitrary player of the coalition $C$, we conclude that $x \in A_{C}^{\prime}$, as desired.

Step 3. Let $N_{a}=\left\{t \in N \mid x_{t} \in A\right\}$. We show that $\mu\left(N_{a}\right)>0$.

If $\mu\left(N_{a}\right)=0$, then $y_{t}=0$ for all $t \in N$. Because all functions $u_{t}$ are positive on the interior of $X$, the inclusion $\operatorname{Int} X \subset A_{t}^{\prime \prime} \subset A_{t}$ holds for each $t \in N$. Thus each alternative in the interior of $X$ is unanimously accepted under the joint strategy $\sigma$. It follows that any player $t \in N \backslash N_{a}$ has a one-shot profitable deviation at any node where $t$ has to make a proposal, namely to propose a point $x \in \operatorname{Int} X$ rather than $x_{t}$. Indeed, the proposal $x_{t}$ of player $t$ is rejected, resulting in the payoff of 0 , while the proposal $x \in \operatorname{Int} X$ is socially accepted and yields a positive payoff.

From $\mu\left(N_{a}\right)>0$ it follows that the set $A$ is non-empty.

Step 4. We now prove that the set $\cap A_{t}^{\prime \prime}$ is non-empty, where the intersection extends over all players $t$ in $N$. It then follows that both sets $A^{\prime \prime}$ and $A^{\prime}$ are intervals.

Let $x$ by the average accepted proposal:

$$
x=\sum_{i \in N_{a}} \mu(i) x_{i} / \mu\left(N_{a}\right) .
$$

By the concavity of the function $u_{t}$

$$
u_{t}(x) \geq \sum_{i \in N_{a}} \mu(i) u_{t}\left(x_{i}\right) / \mu\left(N_{a}\right) \geq \sum_{i \in N_{a}} \mu(i) u_{t}\left(x_{i}\right) /\left[1-\delta \mu\left(N_{r}\right)\right]=y_{t} \geq \delta y_{t},
$$

which shows that $x$ is an element of the set $A_{t}^{\prime}$ for each $t$. If $y_{t}>0$, then the extreme right inequality is strict, so the point $x$ is an element of the set $A_{t}^{\prime \prime}$. And if $y_{t}=0$, the the set $A_{t}^{\prime \prime}$ contains the open interval $\operatorname{Int} X$. Thus the set

$$
\bigcap_{\left\{t \in N \mid y_{t}>0\right\}} A_{t}^{\prime \prime}
$$


is a non-empty open subset of $X$. Any non-empty open subset of $X$ has a non-empty intersection with the interior of $X$, and therefore with the set

$$
\bigcap_{\left\{t \in N \mid y_{t}=0\right\}} A_{t}^{\prime \prime}
$$

We conclude that $\cap A_{t}^{\prime \prime}$ is non-empty.

Step 5. We show that $\left(x_{\bullet}, A_{\bullet}^{\prime}\right)$ is a $\delta$-equilibrium. First we prove that $N_{a}=N$.

Suppose not. Then any player $t \in N \backslash N_{a}$ has a one-shot profitable deviation at any node where player $t$ is a proposer, namely to propose any point $x \in \cap A_{t}^{\prime \prime}$ rather than the point $x_{t}$. Indeed, the proposal $x_{t}$ is rejected, resulting in the payoff of $\delta y_{t}$ for player $t$. The proposal $x$, on the other hand, will be socially accepted, because $x \in \cap A_{t}^{\prime \prime} \subset A^{\prime \prime} \subset A$, resulting in the payoff of $u_{t}(x)$ for player $t$ at node $h$. And, since $x \in A_{t}^{\prime \prime}$, we have the inequality $u_{t}(x)>\delta y_{t}$. This contradicts the assumption that $\sigma$ is a subgame perfect equilibrium.

Since $N_{a}=N$, the expected utility of player $t$ can be written as

$$
y_{t}=\sum_{i \in N} \mu(i) u_{t}\left(x_{i}\right)
$$

Finally, we have to show that the proposal $x_{t}$ of player $t$ maximizes the function $u_{t}$ on $A^{\prime}$. Suppose not. Then there is an $x \in \operatorname{Int} A^{\prime}$ such that $u_{t}(x)>u_{t}\left(x_{t}\right)$. But then player $t$ has a one-shot profitable deviation at any node where player $t$ has to make a proposal, namely to propose the point $x$ rather than the point $x_{t}$. Indeed, the proposal $x$ is socially accepted under the strategy profile $\sigma$, because $x \in \operatorname{Int} A^{\prime} \subset A^{\prime \prime} \subset A$, and it gives a higher payoff to player $t$ than $x_{t}$. This contradicts the hypothesis that $\sigma$ is a subgame perfect equilibrium.

Step 6. Suppose there are players $i^{0}$ and $i^{1}$ such that $\bar{x}_{i^{0}}=0$ and $\bar{x}_{i^{1}}=1$. We show that $A=A^{\prime}$. As Int $A^{\prime} \subset A \subset A^{\prime}$, it suffices to prove that $A$ contains both endpoints of the interval $A^{\prime}$. We already know that $\left(x_{\bullet}, A_{\bullet}^{\prime}\right)$ is a $\delta$-equilibrium. As the ideal point of player $i^{0}$ is zero, the proposal $x_{i}{ }^{0}$ of player $s$ equals the left endpoint of the interval $A^{\prime}$. The ideal point of player $i^{1}$ is one, so $x_{i^{1}}$ equals the right endpoint of the interval $A^{\prime}$. As we have already proved in Step $5, N_{a}=N$, so both $x_{i^{0}}$ and $x_{i^{1}}$ are elements of the set $A$, as desired.

\section{The social acceptance set}

In this section we establish that along any sequence of $\delta$-equilibria, as $\delta$ converges to one, the social acceptance set collapses to a point. The idea of the proof is very simple: we first consider the image $u_{t}(A)$ of the social acceptance set in the utility space. We show that for at least one player $t$ the length of the interval $u_{t}(A)$ is bounded above by $(1-\delta) / \nu$, where $\nu$ depends only on the recognition probabilities. It follows that $u_{t}(A)$ converges to a singleton as $\delta$ goes to one. Strict quasi-concavity of the function $u_{t}$ then implies that 
the set $A$ also converges to a singleton. To highlight the importance of assumption (A2) for this result we consider a world where assumption (A2) is violated and consequently Theorem 3 fails.

At the end of the section a special case of simple majority rule is considered. If the simple majority is required for a passing of a proposal, then the unique bargaining outcome is the ideal point of the median player, i.e. that individual who divides the set of players into two coalitions of equal size, those with lower ideal points and those with higher ideal points. This result has been earlier established in Banks and Duggan [?].

Let $I^{n}$ be a sequence of compact intervals and $I$ be a compact interval. We say that the sequence $I^{n}$ converges to $I$ if the sequence of left endpoints of $I^{n}$ converges to the left endpoint of $I$ and the sequence of right endpoints of $I^{n}$ converges to the right endpoint of $I$. We write Len $(I)$ for the length of an interval $I$, the difference of the upper and lower endpoints. Let $S=\{i \in N \mid \mu(i)>0\}$ and let $\nu=\min _{i \in S}\{\mu(i)\}$.

Proposition 5 Let $a_{\bullet}$ be an element of $[0,1]^{N}$. Let $\delta \in[0,1]$. Suppose that $\delta E\left(a_{\bullet}\right) \leq a_{i}$ for all $i \in S$. Then $a_{i} \leq(1-\delta+\nu \delta) E\left(a_{\bullet}\right) / \nu$ for all $i \in S$.

Proof. For each $i \in S$ we have the following chain of inequalities:

$$
\begin{aligned}
E\left(a_{\bullet}\right) & =\sum_{t \in S} \mu(t) a_{t} \\
& \geq \mu_{i} a_{i}+\left(1-\mu_{i}\right) \delta E\left(a_{\bullet}\right) \\
& =\delta E\left(a_{\bullet}\right)+\mu_{i}\left(a_{i}-\delta E\left(a_{\bullet}\right)\right) \\
& \geq \delta E\left(a_{\bullet}\right)+\nu\left(a_{i}-\delta E\left(a_{\bullet}\right)\right) \\
& =\nu a_{i}+(1-\nu) \delta E\left(a_{\bullet}\right) .
\end{aligned}
$$

Rearranging yields the desired inequality.

We now estimate the length of the image of the social acceptance set in the utility space. Notice that assumption (A2) plays a crucial role in the argument.

Proposition 6 Let $\left(x_{\bullet}, A_{\bullet}\right)$ be a $\delta$-equilibrium inducing the expected payoffs $y_{\bullet}$ and the social acceptance set $A$. Then $\operatorname{Len}\left(u_{t}(A)\right) \leq(1-\delta) / \nu$ for some $t \in S$.

Proof. Let $A=\left[x_{-}, x_{+}\right]$. There exist coalitions $C^{-}$and $C^{+}$in $\mathscr{C}$ such that $x_{-} \in A_{C^{-}}$and $x_{+} \in A_{C^{+}}$. Now, by assumption (A2) there exists a player $t \in C^{-} \cap C^{+}$with $\mu(t)>0$. Thus, both points $x_{-}$and $x_{+}$belong to the individual acceptance set $A_{t}$ of player $t$. Since $A_{t}$ is an interval, it contains the entire social acceptance set $A$. Fix one such player $t$.

We can estimate the utility of player $t$ on the social acceptance set $A$ as follows. Since $A \subset A_{t}$, the utility of player $t$ on the set $A$ is bounded below by $\delta y_{t}$. On the other hand, the proposal $x_{t}$ of player $t$ maximizes player's $t$ utility on the set $A$. Thus

$$
u_{t}(A) \subset\left[\delta y_{t}, u_{t}\left(x_{t}\right)\right] .
$$


Now we apply Proposition 5. Let $a_{i}=u_{t}\left(x_{i}\right)$ for all $i \in N$ and notice that $y_{t}=E\left(a_{\bullet}\right)$. Then we have $a_{i} \geq \delta E\left(a_{\bullet}\right)$ as in Proposition 5 , because each $x_{i}$ is an element of $A$, and therefore an element of $A_{t}$. Proposition 5 implies, in particular, that

$$
u_{t}\left(x_{t}\right) \leq(1-\delta+\nu \delta) y_{t} / \nu .
$$

We can thus estimate the length of the interval $u_{t}(A)$ as follows:

$$
\operatorname{Len}\left(u_{t}(A)\right) \leq(1-\delta+\nu \delta) y_{t} / \nu-\delta y_{t}=(1-\delta) y_{t} / \nu \leq(1-\delta) / \nu,
$$

where the last inequality follows from the fact that $y_{t} \leq 1$. The result follows.

We are now in a position to prove the main result of this section.

Theorem 3 For each natural number n let $A^{n}$ be a social acceptance set in a $\delta^{n}$-equilibrium. If the sequence $A^{n}$ converges to a compact interval $A$ and the sequence $\delta^{n}$ converges to 1 , then $A$ is a singleton.

Proof. Since the set $S$ is finite, we can assume without loss of generality that there exists a player $t$ in $S$ such that

$$
\operatorname{Len}\left(u_{t}\left(A^{n}\right)\right) \leq\left(1-\delta^{n}\right) / \nu
$$

for each $n$. Thus the sequence $\operatorname{Len}\left(u_{t}\left(A^{n}\right)\right)$ converges to zero. Now, $\operatorname{Len}\left(u_{t}(I)\right)$ is a continuous function of the endpoints of the interval $I$. It follows that $\operatorname{Len}\left(u_{t}(A)\right)=0$, i.e. the function $u_{t}$ is constant on the interval $A$. By assumption (A1) the function $u_{t}$ is nonconstant on any non-degenerate interval. It follows that $A$ is a singleton.

We now show, by means of an example, that Theorem 3 need not be true if assumption (A2) is violated. Let the player set $N$ consist of player 0 with the utility function $u_{0}(x)=$ $1-x$, player 1 with the utility function $u_{1}(x)=x$ and a set of players $M$ all having the same utility function $u_{i}(x)=1-|x-1 / 2|$. The utility functions are illustrated in Figure 3. Assumption (A1) is clearly satisfied.

Think of the set $M$ as consisting of a very large number of players. It is then reasonable to assume that there exists a subset $C$ of $M$ such that both $C_{0}=\{0\} \cup C$ and $C_{1}=\{1\} \cup C$ are decisive coalitions. Furthermore, we assume that $\mu(0)=\mu(1)=1 / 2$. Thus only players 0 and 1 ever make proposals. Assumption (A2) is violated because $C_{0} \cap C_{1}=C$ and $\mu(C)=0$.

Now, for each $\delta \in[0,1]$ there exists a $\delta$-equilibrium where $X$ is the social acceptance set. In this equilibrium each player proposes his own ideal point: player 0 proposes 0 , each player in $M$ proposes the point $1 / 2$ and player 1 proposes the point 1 . The expected utility is $1 / 2$ for all players. The individual acceptance set of player 0 is $A_{0}=[0,(1+\delta) / 2]$, the individual acceptance set of player 1 is $A_{1}=[(1-\delta) / 2,1]$ and that of each player in $M$ is $X$. The acceptance set of coalition $C_{0}$ equals the individual acceptance set of player 0 , while the acceptance set of coalition $C_{1}$ equals the individual acceptance set of player 1 . 


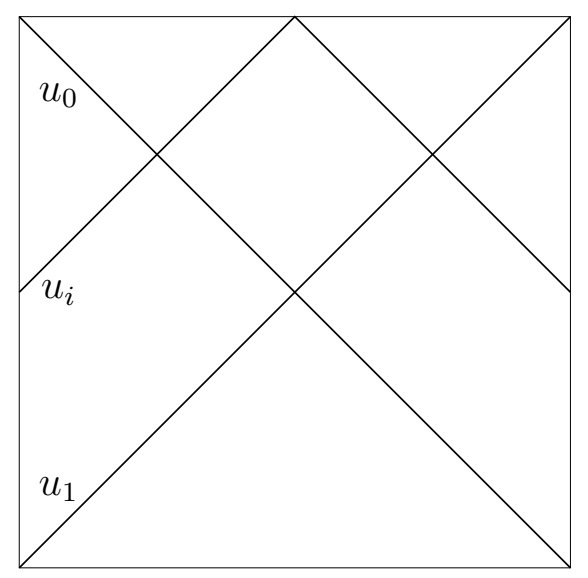

Figure 3: The utility functions in the example of Section 4.

The union of $A_{0}$ and $A_{1}$ is clearly the entire set $X$. Thus the social acceptance set equals $X$.

We conclude this section with two additional results. First we show that each bargaining outcome of the world $\omega$ lies in the interval $[\alpha, \beta]$ as defined below. We also reestablish that, under the simple majority rule, there exists a unique bargaining outcome that only depends on the set of players and their ideal points. The latter result has been established by Banks and Duggan [1], see Theorem 5 and the discussion thereafter.

Define $\alpha$ and $\beta$ as follows:

$$
\alpha=\max _{C \in \mathscr{C}} \min _{t \in C}\left\{\bar{x}_{t}\right\} \text { and } \beta=\min _{C \in \mathscr{C}} \max _{t \in C}\left\{\bar{x}_{t}\right\}
$$

For example, in the case of the unanimity acceptance rule $(\mathscr{C}=\{N\}) \alpha$ equals the minimum of the ideal points and $\beta$ is the maximal ideal point.

Proposition 7 The inequality $\mu\left(\left\{i \in N \mid \alpha \leq \bar{x}_{i} \leq \beta\right\}\right)>0$ holds. In particular, $\alpha \leq \beta$.

Proof. Let $C_{\alpha} \in \mathscr{C}$ and $C_{\beta} \in \mathscr{C}$ be such that

$$
\alpha=\min _{t \in C_{\alpha}}\left\{\bar{x}_{t}\right\} \text { and } \beta=\max _{t \in C_{\beta}}\left\{\bar{x}_{t}\right\}
$$

Thus $\alpha \leq \bar{x}_{t}$ for all $t \in C_{\alpha}$ and $\bar{x}_{t} \leq \beta$ for all $t \in C_{\beta}$. By Assumption (A2) there exists a player $i \in C_{\alpha} \cap C_{\beta}$ such that $\mu(\{i\})>0$. Then $\alpha \leq \bar{x}_{i} \leq \beta$. This proves the result.

Proposition 8 Let $\delta \in[0,1]$. Let $A=\left[x_{-}, x_{+}\right]$be the social acceptance set in $a \delta-$ equilibrium of the world $\omega$. Then $x_{-} \leq \beta$ and $\alpha \leq x_{+}$.

Proof. As before, let $\left[x_{t}^{-}, x_{t}^{+}\right]$be the individual acceptance set of player $t$. The point $\bar{x}_{t}$ is the maximum of the function $u_{t}$ on the set $X$, and so is an element of $A_{t}$. Thus 
$x_{t}^{-} \leq \bar{x}_{t} \leq x_{t}^{+}$for each $t \in N$. Using the formulas for $x_{-}$and $x_{+}$of Section 2, we obtain

$$
\begin{aligned}
& x_{-}=\min _{C \in \mathscr{C}} \max _{t \in C}\left\{x_{t}^{-}\right\} \leq \min _{C \in \mathscr{C}} \max _{t \in C}\left\{\bar{x}_{t}\right\}=\beta, \\
& x_{+}=\max _{C \in \mathscr{C}} \min _{t \in C}\left\{x_{t}^{+}\right\} \geq \max _{C \in \mathscr{C}} \min _{t \in C}\left\{\bar{x}_{t}\right\}=\alpha .
\end{aligned}
$$

Proposition 9 Each bargaining outcome of the world $\omega$ lies in the interval $[\alpha, \beta]$.

Proof. Let $x$ be a bargaining outcome of $\omega$. By Definition 2, the point $x$ is a limit of a sequence $x^{n}$ of points where each $x^{n}$ is an element of the social acceptance set $A^{n}=\left[x_{-}^{n}, x_{+}^{n}\right]$ in a $\delta^{n}$-equilibrium of $\omega$ and the sequence $\delta^{n}$ converges to one. Then by Theorem 3 both sequences $x_{-}^{n}$ and $x_{+}^{n}$ converge to the point $x$. By Proposition $8, x_{-}^{n} \leq \beta$ and $\alpha \leq x_{+}^{n}$ for each $n$. Taking the limit, we obtain the inequalities $x \leq \beta$ and $\alpha \leq x$.

We now turn to a special case of the simple majority rule.

Proposition 10 Consider a world $\omega$ where $|N|=2 n+1$ and the collection $\mathscr{C}$ consists of all coalitions $C \subset N$ with $|C|=n+1$. Then $\alpha=\beta$ is the unique bargaining outcome of the world $\omega$. Furthermore, $\alpha=\beta$ is the unique point $x$ satisfying the inequalities

$$
\left|\left\{i \in N \mid \bar{x}_{i} \leq x\right\}\right| \geq n+1 \text { and }\left|\left\{i \in N \mid x \leq \bar{x}_{i}\right\}\right| \geq n+1 .
$$

Proof. The fact that $\alpha=\beta$ and the inequalities are established by a direct verification. The fact that $\alpha=\beta$ is the unique bargaining outcome follows from Proposition 9 .

For example, if there are seven players four of whom have an ideal point equal to 0 and the remaining three have an ideal point 1 , then 0 is the unique bargaining outcome. If no two players have the same ideal points, then the unique bargaining outcome is an ideal point of the median voter, i.e. that player who divides the set of players into two coalitions of equal size, those with a lower ideal point and those with a higher ideal point. Notice that this point only depends on the collection of players' ideal points. It does not depend on the shape of the utility functions or recognition probabilities.

In the case of the simple majority rule the computation of the bargaining outcome is trivial, because the points $\alpha$ and $\beta$ coincide, so they must be the unique bargaining outcome by Proposition 9. One other special case where $\alpha=\beta$ is when all players' ideal points coincide, in which case $\alpha=\beta$ is the common ideal point and therefore the unique bargaining outcome. In most cases of interest, however, $\alpha<\beta$, and Proposition 9 does not pin down a specific point as a bargaining outcome. To compute the bargaining outcome we rely on a technique based on the characteristic function. 


\section{The characteristic function}

\subsection{The characteristic function and the main result}

Remark 1 Each function $u_{t}$ has left and right derivatives at each point $x$ of the interior of $X$ denoted by $l_{t}(x)$ and $r_{t}(x)$. Both the left and the right derivatives are non-increasing functions of $x$ and $r_{t}(x) \leq l_{t}(x)$. The interval $\left[r_{t}(x), l_{t}(x)\right]$ is the subgradient of $u_{t}$ at point $x$. Given $x \in \operatorname{Int} X$ and $\dot{x} \in X$, the inequality $u_{t}(\dot{x})-u_{t}(x) \leq s(\dot{x}-x)$ holds for each $s \in\left[r_{t}(x), l_{t}(x)\right]$. Furthermore, $r_{t}\left(\bar{x}_{t}\right) \leq 0 \leq l_{t}\left(\bar{x}_{t}\right)$, because $\bar{x}_{t}$ is the maximum of $u_{t}$ on $X$.

For each $x \in \operatorname{Int} X$ we define

$$
\begin{gathered}
\varphi_{-}(x)=\max _{C \in \mathscr{C}} \min _{t \in C}\left\{r_{t}(x) / u_{t}(x), 0\right\} \text { and } \varphi_{+}(x)=\min _{C \in \mathscr{C}} \max _{t \in C}\left\{l_{t}(x) / u_{t}(x), 0\right\}, \\
\mu_{-}(x)=\mu\left(\left\{i \in N \mid x<\bar{x}_{i}\right\}\right) \text { and } \mu_{+}(x)=\mu\left(\left\{i \in N \mid \bar{x}_{i}<x\right\}\right), \\
\xi(x)=\mu_{-}(x) \varphi_{-}(x)+\mu_{+}(x) \varphi_{+}(x) .
\end{gathered}
$$

These are well defined, since each function $u_{t}$ is positive on the interior of the set $X$. The function $\xi$ is referred to as a characteristic function. It will be convenient to extend the characteristic function to $X$ by letting $\xi(0)=+\infty$ and $\xi(1)=-\infty$.

Remark 2 There is nothing particularly important about the use of left derivatives in the function $\varphi_{+}$and right derivatives in $\varphi_{-}$. As the reader can verify, all results remain true if one replaces a derivative $l_{t}(x)$ or $r_{t}(x)$ by an arbitrary element in the subgradient $\left[r_{t}(x), l_{t}(x)\right]$ of the function $u_{t}$.

We now compute the characteristic function in a special case where the utility of an alternative $x$ to player $t$ depends only on the distance between $x$ and player $t$ 's ideal point through a function $h$ that is the same for all players. Let $N$ be a subset of $X$. Suppose that $u_{t}(x)=h(|x-t|)$ where $h:[0,1] \rightarrow[0,1]$ is a decreasing concave continuously differentiable function. Notice that the function $u_{t}$ reaches its maximum on $X$ at the point $t$. Let $g:[0,1] \rightarrow[0,1]$ be defined by $g(x)=h^{\prime}(x) / h(x)$. Then

$$
r_{t}(x) / u_{t}(x)=\left\{\begin{array}{ll}
-g(|x-t|) & \text { if } x<t \\
+g(|x-t|) & \text { if } t \leq x,
\end{array} \quad l_{t}(x) / u_{t}(x)= \begin{cases}-g(|x-t|) & \text { if } x \leq t \\
+g(|x-t|) & \text { if } t<x .\end{cases}\right.
$$

Since $g$ is a non-positive decreasing function, both functions $r_{t}(x) / u_{t}(x)$ and $l_{t}(x) / u_{t}(x)$ are decreasing in the argument $x$ for each $t$ and are increasing in the argument $t$ for each $x$. Using the latter fact we compute:

$$
\begin{gathered}
\varphi_{-}(x)=\max _{C \in \mathscr{C}} \min _{t \in C}\left\{r_{t}(x) / u_{t}(x)\right\}=r_{\alpha}(x) / u_{\alpha}(x), \text { whenever } \varphi_{-}(x)<0, \\
\varphi_{+}(x)=\min _{C \in \mathscr{C}} \max _{t \in C}\left\{l_{t}(x) / u_{t}(x)\right\}=l_{\beta}(x) / u_{\beta}(x), \text { whenever } \varphi_{+}(x)>0,
\end{gathered}
$$


where $\alpha$ and $\beta$ are as in Section 4 . We can restrict our attention to the interval $[\alpha, \beta]$, since we already know that it contains all bargaining outcomes. Since $r_{\alpha}(x) / u_{\alpha}(x)=g(x-\alpha)$ is non-positive and $l_{\beta}(x) / u_{\beta}(x)=-g(\beta-x)$ is non-negative on the interval $[\alpha, \beta]$, we have the following expression for the characteristic function:

$$
\xi(x)=\mu_{-}(x) g(x-\alpha)-\mu_{+}(x) g(\beta-x) \text { for each } \alpha \leq x \leq \beta .
$$

Proposition 11 The characteristic function $\xi$ is a decreasing function on $X$.

Proof. Given a real-valued function $f$ on $X$ let $\operatorname{supp}(f)=\{x \in X|| f(x) \mid>0\}$.

Consider the functions $\varphi_{+}$and $\varphi_{-}$. Clearly, $\varphi_{+}(x) \geq 0$ and $\varphi_{-}(x) \leq 0$ for each $x \in \operatorname{Int} X$. Using the fact that the functions $l_{t}(x)$ and $r_{t}(x)$ are non-increasing, it is easy to show that both $l_{t}(x) / u_{t}(x)$ and $r_{t}(x) / u_{t}(x)$ are decreasing functions on $\operatorname{Int} X$ for each $t$. It then follows that both functions $\varphi_{+}$and $\varphi_{-}$are non-increasing on Int $X$. Furthermore, $\varphi_{+}$is a decreasing function on $\operatorname{supp}\left(\varphi_{+}\right)$and $\varphi_{-}$is a decreasing function on $\operatorname{supp}\left(\varphi_{-}\right)$.

We show that

$$
(0, \beta) \subset \operatorname{supp}\left(\varphi_{+}\right) \subset[0, \beta] .
$$

To prove the first inclusion, let the point $0<x<\beta$ be given. Then for each $C \in \mathscr{C}$ there exists a player $t \in C$ such that $x<\bar{x}_{t}$. For this player $t$ it holds that $r_{t}(x)>0$. It follows that $\varphi_{+}(x)>0$, as desired. To prove the second inclusion let $\beta<x<1$ be given. Then there exists a coalition $C \in \mathscr{C}$ such that for each $t \in C$ we have $\bar{x}_{t}<x$, so $r_{t}(x)<0$. It follows that $\varphi_{+}(x)=0$, as desired. Similarly, one shows that

$$
(\alpha, 1) \subset \operatorname{supp}\left(\varphi_{-}\right) \subset[\alpha, 1]
$$

Consider now the function $\xi$. Write the function $\xi$ as $\xi=\xi_{-}+\xi_{+}$, where $\xi_{-}(x)=$ $\mu_{-}(x) \varphi_{-}(x)$ and $\xi^{+}(x)=\mu_{+}(x) \varphi_{+}(x)$. Of course, $\xi_{-}(x) \leq 0$ and $0 \leq \xi_{+}(x)$ for each $x \in \operatorname{Int} X$.

We show that both functions $\xi_{+}$and $\xi_{-}$are non-increasing on $\operatorname{Int} X$. Indeed, both $\mu_{+}$and $\varphi_{+}$are non-increasing and non-negative on Int $X$. It follows that $\xi_{+}=\mu_{+} \varphi_{+}$is non-increasing. The function $\mu_{-}$is non-decreasing, while $\varphi_{-}$is non-positive and nonincreasing. Therefore, $\xi_{-}=\mu_{-} \varphi_{-}$is a non-increasing function.

Now, $\operatorname{supp}\left(\xi_{+}\right)=\operatorname{supp}\left(\mu_{+}\right) \cap \operatorname{supp}\left(\varphi_{+}\right)$. Since the function $\varphi_{+}$is decreasing on $\operatorname{supp}\left(\varphi_{+}\right)$, the function $\xi_{+}$is decreasing on $\operatorname{supp}\left(\xi_{+}\right) . \operatorname{Similarly,} \operatorname{supp}\left(\xi_{-}\right)=\operatorname{supp}\left(\mu_{-}\right) \cap \operatorname{supp}\left(\varphi_{-}\right)$. Since the function $\varphi_{-}$is decreasing on $\operatorname{supp}\left(\varphi_{-}\right)$, the function $\xi_{-}$is decreasing on $\operatorname{supp}\left(\xi_{-}\right)$.

We now show that $\xi$ is a decreasing function on $\operatorname{Int} X$. Suppose not. Then there exist $0<a<b<1$ such that $\xi(a) \geq \xi(b)$. Because $\xi_{-}$and $\xi_{+}$are non-increasing functions, all three must be constant on the interval $[a, b]$. Because the function $\xi_{-}$is decreasing on the set $\operatorname{supp}\left(\xi_{-}\right)$and the function $\xi_{+}$is decreasing on $\operatorname{supp}\left(\xi_{+}\right)$, both function must be identically equal to zero on the interval $[a, b]$.

In particular, $\xi_{-}(b)=0$ and $\xi_{+}(a)=0$. Now, $\xi_{-}(b)=0$ means that either $\mu_{-}(b)=0$ or $\varphi_{-}(b)=0$. Similarly, $\xi_{+}(a)=0$ means that either $\mu_{+}(a)=0$ or $\varphi_{+}(a)=0$. Now, we cannot have both $\mu_{-}(b)=0$ and $\mu_{+}(a)=0$, because $1=\mu(N) \leq \mu_{-}(b)+\mu_{+}(a)$. Suppose 
$\mu_{+}(a)>0$. Then $\varphi_{+}(a)=0$. Therefore, $\beta \leq a$. But then $\alpha \leq \beta \leq a<b$, so $\varphi_{-}(b)>0$. Therefore, $\mu_{-}(b)=0$. But then $\mu\left(\left\{i \in N \mid \alpha \leq \bar{x}_{i} \leq \beta\right\}=0\right.$, contradicting Proposition 7 .

Finally, $\xi$ is a decreasing function on $X$ because $\xi(0)=+\infty$ and $\xi(1)=-\infty$.

Definition 4 The point $x \in X$ is a generalized zero of the function $f: X \rightarrow \mathbb{R} \cup$ $\{-\infty,+\infty\}$ if there are sequences $x_{-}^{n}$ and $x_{+}^{n}$ of points in $X$ converging to $x$ such that $\lim f\left(x_{-}^{n}\right) \leq 0 \leq \lim f\left(x_{+}^{n}\right)$.

It is clear that any point $x \in X$ such that $f(x)=0$ is a generalized zero of the function $f$ (take $x_{-}^{n}=x$ and $x_{+}^{n}=x$ ). Conversely, if $x$ is a generalized zero of $f$ and $f$ is continuous at point $x$, then $f(x)=0$. A decreasing function $f$ has at most one generalized zero. We are now in a position to state the main result of the paper.

Theorem 4 (Main result) Each world $\omega$ has a unique bargaining outcome. The bargaining outcome of $\omega$ is the unique generalized zero of the characteristic function $\xi$.

\subsection{Some intuition}

In this subsection we wish to argue that the bargaining outcome is in some sense robust to renegotiations, when it is taken to be a status quo. All derivations are deliberately informal, the purpose being to provide some intuition behind, rather than the proof of, the main result.

Suppose that there has been a preliminary stage of negotiations that resulted in the agreement to implement some alternative $x$ at the moment of time $t>0$. Imagine now that the players negotiate again over the incremental change in the outcome together with an incremental reduction in length of delay, with the status quo being the implementation of the alternative $x$ at time $t$. Let $d x$ be an incremental change in the outcome and $-d t<0$ be an incremental reduction in length of delay. The ratio $d x / d t$ can be though of the as the speed of the adjustment. A player $i$ evaluates the resulting time-outcome pair using the function $U_{i}(x, t)=\delta^{t} u_{i}(x)$. Obviously, each player prefers a change away from $x$ in the direction of its own ideal point, however, a player also accepts an incremental change in the opposite direction, given a sufficiently large reduction in the length of delay. The proposal $(d x,-d t)$ is accepted by player $i$ if results in a non-negative change in the utility $U_{i}$, so the condition for the acceptance of $(d x,-d t)$ is that

$$
d U_{i}(x, t)=u_{i}^{\prime}(x) d x-\ln (\delta) u_{i}(x) d t \geq 0 .
$$

Player $i$ whose ideal point is to the left of the point $x$ accepts a pair $(d x,-d t)$ provided that $d x / d t \leq \ln (\delta) u_{i}(x) / u_{i}^{\prime}(x)$. Similarly, a player $i$ whose ideal point is to the right of $x$ any pair $(d x,-d t)$ provided that $d x / d t \geq \ln (\delta) u_{i}(x) / u_{i}^{\prime}(x)$. The acceptance set of coalition $C$ is therefore an interval with the endpoints

$$
\phi_{C}^{-}(x)=\max _{\substack{i \in C \\ x<\bar{x}_{i}}}\left\{\frac{\ln (\delta) u_{i}(x)}{u_{i}^{\prime}(x)},-\infty\right\} \text { and } \phi_{C}^{+}(x)=\min _{\substack{i \in C \\ \bar{x}_{i}<x}}\left\{\frac{\ln (\delta) u_{i}(x)}{u_{i}^{\prime}(x)},+\infty\right\} .
$$


The social acceptance set is an interval with the endpoints

$$
\phi_{-}(x)=\min _{C \in \mathscr{C}} \phi_{C}^{-}(x) \text { and } \phi_{+}(x)=\max _{C \in \mathscr{C}} \phi_{C}^{+}(x) .
$$

Each player with an ideal point to the left of $x$ proposes the left endpoint of the social acceptance set, and a player with an ideal point to the right of $x$ proposes the right endpoint of the social acceptance set. Any player whose ideal point is $x$ makes a proposal $d x / d t=0$. Thus the average proposed speed is

$$
\psi(x)=\mu_{-}(x) \phi_{-}(x)+\mu_{+}(x) \phi_{+}(x) .
$$

In fact, the function $\psi$ is related to the function $\xi$ through the equation $\varphi_{-} \varphi_{+} \psi=\ln (\delta) \xi$. To see this, notice that

$$
\begin{gathered}
\phi_{C}^{-}(x)=\ln (\delta) / \max _{i \in C}\left\{u_{i}^{\prime}(x) / u_{i}(x), 0\right\} \text { and } \phi_{C}^{+}(x)=\ln (\delta) / \min _{i \in C}\left\{u_{i}^{\prime}(x) / u_{i}(x), 0\right\} \\
\phi_{-}(x)=\ln (\delta) / \varphi_{+}(x) \text { and } \phi_{C}^{+}(x)=\ln (\delta) / \varphi_{-}(x) .
\end{gathered}
$$

Assuming that $\varphi_{-}$is negative and $\varphi_{+}$is positive on the relevant interval, every zero of $\xi$ is also a zero of $\psi$, and vice versa.

Now, it seems very intuitive that the bargaining outcome should be a zero of the function $\psi$. Indeed, a zero of the function $\psi$ can be thought of being robust to a renegotiation, when taken as a status quo, in the sense that the average proposed incremental change is zero. Alternatively, one can view a zero of $\psi$ as a rest point of a dynamics $d x / d t=\psi(x)$ that adjusts the outcome in the direction of the average proposal.

\section{The proof of the main result}

The central idea of the proof is to estimate the values of the characteristic function on the endpoints of the social acceptance set. Thus the main result of this section is Theorem 5 which establishes the inequalities $\xi\left(x_{-}\right) \geq 0$ and $0 \geq \xi\left(x_{+}\right)$, where $x_{-}$and $x_{+}$are the endpoints of the social acceptance set in a $\delta$-equilibrium for any $\delta<1$. The inequalities imply immediately that any bargaining outcome is a generalized zero of the function $\xi$, and, since $\xi$ is a decreasing function, that the bargaining outcome is unique. As a byproduct of Theorem 5 we also obtain an interesting fact that the bargaining outcome is an element of the social acceptance set of any $\delta$-equilibrium for any $\delta \in[0,1)$.

The following proposition is a decisive step of the proof.

Proposition 12 Suppose $\delta \in[0,1)$. Let $\left(x_{\bullet}, A_{\bullet}\right)$ be a $\delta$-equilibrium inducing the expected payoffs $y_{\bullet}$, the acceptance set $A_{C}$ for each coalition $C$ and the social acceptance set $A=$ $\left[x_{-}, x_{+}\right]$. Let

$$
d_{-}=\frac{x_{-}-E\left(x_{\bullet}\right)}{1-\delta} \text { and } d_{+}=\frac{x_{+}-E\left(x_{\bullet}\right)}{1-\delta}
$$


If $x_{-} \in \operatorname{Int} X$, then the following inequalities hold:

$$
\begin{aligned}
& -1 \leq \delta d_{+} \varphi_{-}\left(x_{-}\right), \\
& -1 \geq \delta d_{-} \varphi_{+}\left(x_{-}\right) .
\end{aligned}
$$

If $x_{+} \in \operatorname{Int} X$, then the following inequalities hold:

$$
\begin{aligned}
& -1 \leq \delta d_{-} \varphi_{+}\left(x_{+}\right), \\
& -1 \geq \delta d_{+} \varphi_{-}\left(x_{+}\right) .
\end{aligned}
$$

Proof. We prove the first inequality. Assume that $x_{-} \in \operatorname{Int} X$.

Let $t$ be an arbitrary player. For each $i \in N$ we have the inequalities

$$
u_{t}\left(x_{+}\right)-u_{t}\left(x_{i}\right) \leq r_{t}\left(x_{i}\right)\left[x_{+}-x_{i}\right] \leq r_{t}\left(x_{-}\right)\left[x_{+}-x_{i}\right],
$$

where the first inequality is a defining inequality for the subgradient of the function $u_{t}$, and the second inequality follows from the fact that the left derivative is a non-increasing function, so that $r_{t}\left(x_{i}\right) \leq r_{t}\left(x_{-}\right)$for each $i \in N$ and the fact that $0 \leq x_{+}-x_{i}$. (The right derivative at the point $x_{i}$ exists if $x_{i}<x_{+}$, because in that case $x_{i}$ is in the interior of $X$. If $x_{i}=x_{+}$, then the extreme left and right hand sides of the above inequality are both zero.) Taking the expected value with respect to $i$ in both sides of the above inequality yields

$$
u_{t}\left(x_{+}\right)-y_{t} \leq r_{t}\left(x_{-}\right)\left[x_{+}-E\left(x_{\bullet}\right)\right] .
$$

Let $C_{+} \in \mathscr{C}$ be such that $x_{+} \in A_{C_{+}}$. Then $\delta y_{t} \leq u_{t}\left(x_{+}\right)$for each $t \in C$. Let $t$ be a player in the coalition $C$. Therefore,

$$
\begin{aligned}
0 & \leq u_{t}\left(x_{+}\right)-\delta y_{t}= \\
& =(1-\delta) u_{t}\left(x_{+}\right)+\delta\left[u_{t}\left(x_{+}\right)-y_{t}\right] \leq \\
& \leq(1-\delta) u_{t}\left(x_{+}\right)+\delta r_{t}\left(x_{-}\right)\left[x_{+}-E\left(x_{\bullet}\right)\right] .
\end{aligned}
$$

Dividing by $(1-\delta)$ yields

$$
0 \leq u_{t}\left(x_{+}\right)+\delta r_{t}\left(x_{-}\right) d_{+} .
$$

Now, either $\bar{x}_{t} \leq x_{-}$or $x_{-}<\bar{x}_{t}$. If the former, then then the function $u_{t}$ is non-increasing on the interval $\left[x_{-}, x_{+}\right]$, in particular $u_{t}\left(x_{+}\right) \leq u_{t}\left(x_{-}\right)$. If the latter, then $0 \leq r_{t}\left(x_{-}\right)$. In either case we obtain the inequality

$$
0 \leq u_{t}\left(x_{-}\right)+\delta r_{t}\left(x_{-}\right) d_{+} .
$$

Rearranging yields the inequality

$$
-1 \leq \delta d_{+} r_{t}\left(x_{-}\right) / u_{t}\left(x_{-}\right) .
$$

Since $t$ is arbitrary member of coalition $C_{+}$, we have

$$
-1 \leq \min _{t \in C_{+}}\left\{\delta d_{+} r_{t}\left(x_{-}\right) / u_{t}\left(x_{-}\right), 0\right\} .
$$


Therefore,

$$
\begin{aligned}
-1 & \leq \max _{C \in \mathscr{C}} \min _{t \in C}\left\{\delta d_{+} r_{t}\left(x_{-}\right) / u_{t}\left(x_{-}\right), 0\right\}= \\
& =\max _{C \in \mathscr{C}} \min _{t \in C}\left\{r_{t}\left(x_{-}\right) / u_{t}\left(x_{-}\right), 0\right\} \delta d_{+}= \\
& =\varphi_{-}\left(x_{-}\right) \delta d_{+} .
\end{aligned}
$$

The proof of the third inequality is a jigged-jagged version of the proof of the first one. Assume that $x_{+} \in \operatorname{Int} X$. Let $C_{-} \in \mathscr{C}$ be such that $x_{-} \in A_{C_{-}}$. Replacing every instance where $x_{-}$occurs by $x_{+}$and vice versa, one shows that

$$
-1 \leq \delta d_{-} l_{t}\left(x_{+}\right) / u_{t}\left(x_{+}\right)
$$

for each $t \in C_{-}$. Therefore,

$$
-1 \leq \min _{t \in C_{-}}\left\{\delta d_{-} l_{t}\left(x_{+}\right) / u_{t}\left(x_{+}\right), 0\right\} .
$$

Taking into account that $d_{-} \leq 0$, we then obtain

$$
\begin{aligned}
-1 & \leq \max _{C \in \mathscr{C}} \min _{t \in C}\left\{\delta d_{-} l_{t}\left(x_{+}\right) / u_{t}\left(x_{+}\right), 0\right\}= \\
& =\min _{C \in \mathscr{C}} \max _{t \in C}\left\{l_{t}\left(x_{+}\right) / u_{t}\left(x_{+}\right), 0\right\} \delta d_{-}= \\
& =\varphi_{+}\left(x_{+}\right) \delta d_{-} .
\end{aligned}
$$

We prove the fourth inequality. Assume that $x_{+} \in \operatorname{Int} X$. Let $C$ be an arbitrary coalition from $\mathscr{C}$. First we argue that there exists a player $t \in C$ such that $u_{t}\left(x_{+}\right) \leq \delta y_{t}$. Suppose not. Then $\delta y_{t}<u_{t}\left(x_{+}\right)$for each $t \in C$. Then the acceptance set $A_{C}$ of coalition $C$ contains an open neighborhood of the point $x_{+}$, and so does the social acceptance set $A$. But $x_{+}$is an interior point of $X$, so any open neighborhood of $x_{+}$contains a point $x>x_{+}$. This contradicts the fact that $x_{+}$is the right endpoint of the interval $A$.

Fix any player $t \in C$ such that $u_{t}\left(x_{+}\right) \leq \delta y_{t}$. For each $i \in N$ we have the inequality

$$
u_{t}\left(x_{i}\right)-u_{t}\left(x_{+}\right) \leq r_{t}\left(x_{+}\right)\left[x_{i}-x_{+}\right]
$$

Taking the expected values on both sides with respect to $i$ yields

$$
u_{t}\left(x_{+}\right)-y_{t} \geq r_{t}\left(x_{+}\right)\left[x_{+}-E\left(x_{\bullet}\right)\right] .
$$

Therefore,

$$
\begin{aligned}
0 & \geq u_{t}\left(x_{+}\right)-\delta y_{t}= \\
& =(1-\delta) u_{t}\left(x_{+}\right)+\delta\left[u_{t}\left(x_{+}\right)-y_{t}\right] \geq \\
& \geq(1-\delta) u_{t}\left(x_{+}\right)+\delta r_{t}\left(x_{+}\right)\left[x_{+}-E\left(x_{\bullet}\right)\right]
\end{aligned}
$$

Dividing by $1-\delta$ gives

$$
0 \geq u_{t}\left(x_{+}\right)+\delta r_{t}\left(x_{+}\right) d_{+}
$$


Rearranging yields the inequality

$$
-1 \geq \delta d_{+} r_{t}\left(x_{+}\right) / u_{t}\left(x_{+}\right) .
$$

Thus,

$$
-1 \geq \min _{i \in C}\left\{\delta d_{+} r_{i}\left(x_{+}\right) / u_{i}\left(x_{+}\right), 0\right\} .
$$

Since $C$ is an arbitrary element of the collection $\mathscr{C}$, we obtain

$$
\begin{aligned}
-1 & \geq \max _{C \in \mathscr{C}} \min _{i \in C}\left\{\delta d_{+} r_{i}\left(x_{+}\right) / u_{i}\left(x_{+}\right), 0\right\}= \\
& =\max _{C \in \mathscr{C}} \min _{i \in C}\left\{r_{i}\left(x_{+}\right) / u_{i}\left(x_{+}\right), 0\right\} \delta d_{+}= \\
& =\varphi_{-}\left(x_{+}\right) \delta d_{+} .
\end{aligned}
$$

The proof of the second inequality is similar to the proof of the fourth inequality. Assume that $x_{-} \in \operatorname{Int} X$. Replacing every instance where $x_{-}$occurs by $x_{+}$and vice versa, one shows that for each coalition $C \in \mathscr{C}$ there exists a player $t \in C$ such that

$$
-1 \geq \delta d_{-} l_{t}\left(x_{-}\right) / u_{t}\left(x_{-}\right)
$$

Thus,

$$
-1 \geq \min _{i \in C}\left\{\delta d_{-} l_{i}\left(x_{-}\right) / u_{i}\left(x_{-}\right), 0\right\} .
$$

Since $C$ is an arbitrary element of the collection $\mathscr{C}$, and taking into account that $d_{-} \leq 0$, we then obtain

$$
\begin{aligned}
-1 & \geq \max _{C \in \mathscr{C}} \min _{i \in C}\left\{\delta d_{-} l_{i}\left(x_{-}\right) / u_{i}\left(x_{-}\right), 0\right\}= \\
& =\min _{C \in \mathscr{C}} \max _{i \in C}\left\{l_{i}\left(x_{-}\right) / u_{i}\left(x_{-}\right), 0\right\} \delta d_{-}= \\
& =\varphi_{+}\left(x_{-}\right) \delta d_{-} .
\end{aligned}
$$

This completes the proof.

Proposition 13 Suppose $\delta \in[0,1)$. Let $\left(x_{\bullet}, A_{\bullet}\right)$ be a $\delta$-equilibrium inducing the social acceptance set $A=\left[x_{-}, x_{+}\right]$. Let

$$
d=\frac{x_{+}-x_{-}}{1-\delta}
$$

If $x_{-} \in \operatorname{Int} X$, then, the following inequalities hold:

$$
\begin{aligned}
-1 & \leq \delta d \mu_{-}\left(x_{-}\right) \varphi_{-}\left(x_{-}\right), \\
1 & \leq \delta d \mu_{+}\left(x_{-}\right) \varphi_{+}\left(x_{-}\right) .
\end{aligned}
$$

If $x_{+} \in \operatorname{Int} X$, then the following inequalities hold:

$$
\begin{aligned}
1 & \geq \delta d \mu_{+}\left(x_{+}\right) \varphi_{+}\left(x_{+}\right) \\
-1 & \geq \delta d \mu_{-}\left(x_{+}\right) \varphi_{-}\left(x_{+}\right) .
\end{aligned}
$$


Proof. Let $d_{-}$and $d_{+}$be as in Proposition 12. We use the following estimates of $E\left(x_{\bullet}\right)$ :

$$
\begin{aligned}
& E\left(x_{\bullet}\right) \leq \mu\left(\bar{x}_{i}<x_{-}\right) x_{-}+\mu\left(x_{-} \leq \bar{x}_{i}\right) x_{+}, \\
& E\left(x_{\bullet}\right) \leq \mu\left(\bar{x}_{i} \leq x_{-}\right) x_{-}+\mu\left(x_{-}<\bar{x}_{i}\right) x_{+}, \\
& E\left(x_{\bullet}\right) \geq \mu\left(\bar{x}_{i} \leq x_{+}\right) x_{-}+\mu\left(x_{+}<\bar{x}_{i}\right) x_{+}, \\
& E\left(x_{\bullet}\right) \geq \mu\left(\bar{x}_{i}<x_{+}\right) x_{-}+\mu\left(x_{+} \leq \bar{x}_{i}\right) x_{+},
\end{aligned}
$$

where $\mu\left(\bar{x}_{i}<x_{-}\right)$stands for $\mu\left(\left\{i \in N \mid \bar{x}_{i}<x_{-}\right\}\right)$, e.t.c. To derive the first of the estimates, let $z_{i}$ be equal to $x_{-}$whenever $\bar{x}_{i}<x_{-}$and $x_{+}$otherwise. Then $x_{i} \leq z_{i}$ for each $i \in N$. Therefore, $E\left(x_{\bullet}\right) \leq E\left(z_{\bullet}\right)$. Other estimates are derived similarly.

Use the estimates for $E\left(x_{\bullet}\right)$ to obtain, respectively, the following inequalities:

$$
\begin{aligned}
& x_{+}-E\left(x_{\bullet}\right) \geq x_{+}-\left[\mu\left(\bar{x}_{i}<x_{-}\right) x_{-}+\mu\left(x_{-} \leq \bar{x}_{i}\right) x_{+}\right]=\mu\left(\bar{x}_{i}<x_{-}\right)\left[x_{+}-x_{-}\right], \\
& x_{-}-E\left(x_{\bullet}\right) \geq x_{-}-\left[\mu\left(\bar{x}_{i} \leq x_{-}\right) x_{-}+\mu\left(x_{-}<\bar{x}_{i}\right) x_{+}\right]=-\mu\left(x_{-}<\bar{x}_{i}\right)\left[x_{+}-x_{-}\right], \\
& x_{-}-E\left(x_{\bullet}\right) \leq x_{-}-\left[\mu\left(\bar{x}_{i} \leq x_{+}\right) x_{-}+\mu\left(x_{+}<\bar{x}_{i}\right) x_{+}\right]=-\mu\left(x_{+}<\bar{x}_{i}\right)\left[x_{+}-x_{-}\right], \\
& x_{+}-E\left(x_{\bullet}\right) \leq x_{+}-\left[\mu\left(\bar{x}_{i}<x_{+}\right) x_{-}+\mu\left(x_{+} \leq \bar{x}_{i}\right) x_{+}\right]=\mu\left(\bar{x}_{i}<x_{+}\right)\left[x_{+}-x_{-}\right] .
\end{aligned}
$$

Dividing by $1-\delta$ gives

$$
\begin{aligned}
& d_{+} \geq \mu\left(\bar{x}_{i}<x_{-}\right) d, \\
& d_{-} \geq-\mu\left(x_{-}<\bar{x}_{i}\right) d, \\
& d_{-} \leq-\mu\left(x_{+}<\bar{x}_{i}\right) d, \\
& d_{+} \leq \mu\left(\bar{x}_{i}<x_{+}\right) d .
\end{aligned}
$$

Now each inequality of Proposition 13 is obtained by combining the respective inequality of Proposition 12 with the respective inequality above.

$$
\begin{aligned}
& -1 \leq \delta d_{+} \varphi_{-}\left(x_{-}\right) \leq \delta \mu\left(\bar{x}_{i}<x_{-}\right) d \varphi_{-}\left(x_{-}\right)=\delta d \mu_{-}\left(x_{-}\right) \varphi_{-}\left(x_{-}\right), \\
& -1 \geq \delta d_{-} \varphi_{+}\left(x_{-}\right) \geq-\delta \mu\left(x_{-}<\bar{x}_{i}\right) d \varphi_{+}\left(x_{-}\right)=-\delta d \mu_{+}\left(x_{-}\right) \varphi_{+}\left(x_{-}\right), \\
& -1 \leq \delta d_{-} \varphi_{+}\left(x_{+}\right) \leq-\delta \mu\left(x_{+}<\bar{x}_{i}\right) d \varphi_{+}\left(x_{+}\right)=-\delta d \mu_{+}\left(x_{+}\right) \varphi_{+}\left(x_{+}\right), \\
& -1 \geq \delta d_{+} \varphi_{-}\left(x_{+}\right) \geq \delta \mu\left(\bar{x}_{i}<x_{+}\right) d \varphi_{-}\left(x_{+}\right)=\delta d \mu_{-}\left(x_{+}\right) \varphi_{-}\left(x_{+}\right) .
\end{aligned}
$$

Of course, the first two inequalities are obtained under the assumption that $x_{-} \in \operatorname{Int} X$ and the last two under the assumption that $x_{+} \in \operatorname{Int} X$.

Proposition 14 below strengthens Proposition 1 in Section 2. While Proposition 1 establishes that the social acceptance set is an interval containing the average proposal, the Proposition 14 states that it contains the average proposal together an open neighborhood.

Proposition 14 Suppose $\delta \in[0,1)$ and let $\left(x_{\bullet}, A_{\bullet}\right)$ be a a $\delta$-equilibrium inducing the expected payoffs $y_{\bullet}$ and the social acceptance set $A$. Then $A$ contains an open neighborhood of the point $E\left(x_{\bullet}\right)$. 
Proof. Each individual acceptance set $A_{t}$ contains the point $E\left(x_{\bullet}\right)$ by the concavity of the function $u_{t}$. Indeed,

$$
u_{t}\left(E\left(x_{\bullet}\right)\right) \geq E\left(u_{t}\left(x_{\bullet}\right)\right)=y_{t} \geq \delta y_{t} .
$$

Furthermore, if $y_{t}>0$, the last inequality is strict, so the point $E\left(x_{\bullet}\right)$ is contained in the interior of $A_{t}$. If $y_{t}=0$, then the set $A_{t}$ contains the interval $(0,1)$. Thus, in either case, the set $A_{t}$ contains the point $E\left(x_{\bullet}\right)$ together with an open neighborhood. It follows that for each coalition $C$ the acceptance set $A_{C}$ contains an open neighborhood of the point $E\left(x_{\bullet}\right)$. The result follows.

Theorem 5 Suppose $\delta \in[0,1)$ and let $A=\left[x_{-}, x_{+}\right]$be the social acceptance set in a $\delta$-equilibrium of $\omega$. Then $\xi\left(x_{-}\right) \geq 0 \geq \xi\left(x_{+}\right)$.

Proof. By Proposition 14 the the set $A$ is non-degenerate. In particular, $x_{-}<1$. If $0<x_{-}<1$, then $\xi\left(x_{-}\right) \geq 0$ as can be seen by adding up the first two inequalities of Proposition 13 and dividing by $\delta d, d$ being non-zero again by Proposition 14 . If $x_{-}=0$, then $\xi\left(x_{-}\right)=+\infty$ by the definition of $\xi$. Similarly, $x_{+}>0$ by Proposition 14. If $0<x_{+}<1$, then $\xi\left(x_{+}\right) \leq 0$ is obtained by adding up the last two inequalities of Proposition 13 and dividing by $\delta d$. And if $x_{+}=1$, then $\xi\left(x_{+}\right)=-\infty$ by the definition of $\xi$.

Proposition 15 Each bargaining outcome of the world $\omega$ is a generalized zero point of the characteristic function $\xi$.

Proof. Let $x$ be a bargaining outcome of $\omega$. By Definition 2, there exist sequences $A^{n}$, $\delta^{n}$, and $x^{n}$ such that $A^{n}$ is a social acceptance set in a $\delta^{n}$-equilibrium of $\omega, x^{n}$ is a point in $A^{n}$, the sequence $\delta^{n}$ converges to 1 and $x^{n}$ converges to $x$. Let $A^{n}=\left[x_{-}^{n}, x_{+}^{n}\right]$. Replacing, if necessary, sequences by subsequences, assume that both $x_{-}^{n}$ and $x_{+}^{n}$ converge. Then Theorem 3 implies that both sequences converge to $x$. Theorem 5 implies that $\lim \xi\left(x_{-}^{n}\right) \geq 0 \geq \lim \xi\left(x_{+}^{n}\right)$, as desired.

The following theorem follows immediately from Theorem 5 and Proposition 15.

Theorem 6 Let $x$ be a bargaining outcome of the world $\omega$. Suppose $\delta \in[0,1)$ and let $A$ be the social acceptance set in a $\delta$-equilibrium of $\omega$. Then $x$ is an element of $A$.

\section{Appendix}

We now provide a proof of Proposition 4. To prove the proposition we introduce a lexicographic order on the collection $\mathscr{C}$ of decisive coalitions induced by the order $>$ on the set $N$ of players. Recall that $>$ denotes the response sequence in the game $\Gamma$. 
Definition 5 Given a natural number $k$ and a coalition $C \in \mathscr{C}$ let $\ell_{k}(C)$ be the $k$-th lowest element of the coalition $C$, in the order $>$ (we let $\ell_{k}(C)=\ell_{|C|}(C)$ for each $k>|C|$ ). Define an lexicographic order $>$ on the set $\mathscr{C}$ by letting $C_{1}>C_{2}$ whenever there exists an natural number $K$ such that $\ell_{k}\left(C_{1}\right)=\ell_{k}\left(C_{2}\right)$ for each $k=1, \ldots, K$ and $\ell_{K}\left(C_{1}\right)>\ell_{K}\left(C_{2}\right)$.

To compare the coalition $C_{1}$ to the coalition $C_{2}$ we first compare their minimal elements. If the lowest element of $C_{1}$ is bigger than the lowest element of $C_{2}$, than $C_{1}>C_{2}$. And if the lowest element of $C_{1}$ equals the lowest element of $C_{2}$, we go on to compare the second lowest elements. If the second lowest element of $C_{1}$ is bigger than that of $C_{2}$, then $C_{1}>C_{2}$. If the second lowest elements of the two coalitions are also the same, we go on to compare the third lowest elements, and so on. For example, take $C_{1}=\{1,2,4,5\}$ and $C_{2}=\{1,2,3,6,7\}$ and let the order on the set $N$ be the natural order $(1<2<\cdots<7)$. Then $C_{1}>C_{2}$.

Clearly, the lexicographic order $>$ is a strict total order on $\mathscr{C}$. We shall be interested in the maximal elements of $>$ on a subset $\mathscr{C}^{\prime}$ of $\mathscr{C}$. For each $\mathscr{C}^{\prime} \subset \mathscr{C}$ there is a unique maximal element of $>$ on $\mathscr{C}^{\prime}$. For the rest of this section we assume that $\mathscr{C}$ only includes the minimal decisive coalitions, i.e. there are no $C_{1}$ and $C_{2}$ in $\mathscr{C}$ such that $C_{1} \subset C_{2}$.

Proposition 16 Let $\mathscr{C}^{\prime} \subset \mathscr{C}$ and let $C \in \mathscr{C}^{\prime}$ be the unique maximal element of $>$ on $\mathscr{C}^{\prime}$. Then for each $t \in C$ and each $C^{\prime} \in \mathscr{C}^{\prime}$ either $t \in C^{\prime}$ or there is a player $i \in C^{\prime} \backslash C$ such that $t>i$.

Proof. Let $t \in C$ and $C^{\prime} \in \mathscr{C}^{\prime}$. Since $C>C^{\prime}$, there exists a $K$ such that $\ell_{k}(C)=\ell_{k}\left(C^{\prime}\right)$ for each $k=1, \ldots, K$ and $\ell_{K}(C)>\ell_{K}\left(C^{\prime}\right)$. If $t \leq \ell_{K}(C)$, then $t=\ell_{k}(C)$ for some $k=1, \ldots, K$, in which case $t=\ell_{k}\left(C^{\prime}\right)$ and so is an element of $C^{\prime}$.

Suppose now that $t>\ell_{K}(C)$ and let $i=\ell_{K}\left(C^{\prime}\right)$. We know that $t>i$, and that the player $i$ is an element of $C^{\prime}$. It remains to show that $i$ is not an element of $C$. Suppose it is. Then $\ell_{K}(C)>i$, so there is some $J<K$ such that $\ell_{J}(C)=i$. But we know that $\ell_{J}(C)=\ell_{J}\left(C^{\prime}\right)$. Thus $\ell_{K}\left(C^{\prime}\right)=i=\ell_{J}\left(C^{\prime}\right)$. This is possible only if $\left|C^{\prime}\right| \leq J$. But then each element of $C^{\prime}$ can be written as $\ell_{j}\left(C^{\prime}\right)$ for some $j=1, \ldots, J$, which equals the element $\ell_{j}(C)$ of the set $C$. Thus $C^{\prime} \subset C$, a contradiction.

Proposition 4 Let $\sigma=\left(x_{\bullet}, A_{\bullet}\right)$ be a joint stationary strategy in the game $\Gamma$ inducing the acceptance set $A_{C}$ for coalition $C$ and the social acceptance set $A$. Let $x \in X$.

(a) Suppose $x$ is not an element of $A$. Then for each $C \in \mathscr{C}$ there is a player $t \in C$ such that $x$ is not an element of the set $A_{t}$ and player t's vote is pivotal at some node $h$ of the game $\Gamma$.

(b) Suppose $x$ is an element of $A$. Then there exists a $C \in \mathscr{C}$ such that $x$ is an element of $A_{C}$ and for each $t \in C$ player t's vote is pivotal at some node $h$ of the game $\Gamma$.

Proof. We prove part (a). Fix a coalition $C^{\prime \prime} \in \mathscr{C}$. We show $C^{\prime \prime}$ contains a player $t$ whose vote is pivotal at some node $h$ of the game $\Gamma$. 
Given a coalition $C^{\prime} \in \mathscr{C}$, let $C_{r}^{\prime}=\left\{i \in C^{\prime} \mid x \notin A_{i}\right\}$. The set $C_{r}^{\prime}$ is non-empty for each $C^{\prime} \in \mathscr{C}$. Choose a coalition $C \in \mathscr{C}$ having the following properties: (a) $C_{r} \subset C^{\prime \prime}$ and (b) there is no coalition $C^{\prime} \in \mathscr{C}$ such that $C_{r}^{\prime}$ is a strict subset of $C_{r}$. Clearly, such a coalition $C$ exists: the set $C_{r}$ is a minimal element of the collection $\left\{C_{r}^{\prime} \mid C^{\prime} \in \mathscr{C}: C_{r}^{\prime} \subset C^{\prime \prime}\right\}$ of sets ordered by inclusion. Let $t$ be the last player of the set $C_{r}$ in the response order $>$. Notice that $t$ is an element of $C^{\prime \prime}$ and that $x$ is not an element of $A_{t}$.

Let $h$ be any node of the game $\Gamma$ where (a) player $t$ reacts to the proposal $x$, (b) each player $i \in C$ preceding player $t$ in the response order $>$ accepts the proposal $x$, (c) each player $i \in N \backslash C$ preceding player $t$ in the response order $>$ rejects the proposal $x$. As follows from the considerations below, the vote of player $t$ at the node $h$ is decisive: the proposal $x$ is socially accepted if and only if it is accepted by player $t$.

Notice that under the strategy $\sigma_{t}$ player $t$ rejects the proposal $x$. We now show that the proposal $x$ is turned down on the path of play induced by the joint strategy $\sigma$ in the subgame $\Gamma(h)$. Indeed, coalition $C$ rejects the proposal $x$, because player $t \in C$ rejects $x$ at the node $h$. Take any $C^{\prime} \in \mathscr{C}$ other than $C$. By the choice of the coalition $C$, there is a player $i \in C_{r}^{\prime} \backslash C_{r}$. If $i=t$ or $i>t$, player $i$ rejects the proposal $x$ along the path of play induced by the joint strategy $\sigma$, because $x \notin A_{i}$. And if $i<t$, then player $i$ rejects the proposal $x$ at the node $h$ by condition (c). Notice that player $i$ is not an element of the coalition $C$, for otherwise the inclusions $i \in C$ and $i \in C_{r}^{\prime}$ would imply the inclusion $i \in C_{r}$, leading to a contradiction.

Consider now a strategy $\sigma_{t}^{\prime}$ for player $t$ that coincides with $\sigma_{t}$ on all nodes but $h$, where it accepts the proposal $x$. We show that the proposal $x$ is accepted by the coalition $C$ on the path of play induced by the joint strategy $\left(\sigma_{t}^{\prime}, \sigma_{-t}\right)$ in the subgame $\Gamma(h)$. Since $t$ is chosen to be the last player of $C_{r}$ in the response sequence, each member $i$ of the coalition $C$ following player $t$ in the response order accepts the proposal $x$ on the path of play induced by $\left(\bar{\sigma}_{t}, \sigma_{-t}\right)$. And each member of coalition $C$ preceding player $t$ in the response order accepts $x$ at the node $h$ by condition (b).

We now prove part (b) of the proposition. As $x$ is assumed an element of the set $A$, the collection $\mathscr{C}^{\prime}=\left\{C \in \mathscr{C} \mid x \in A_{C}\right\}$ is non-empty. Now choose a coalition $C \in \mathscr{C}^{\prime}$ having the following property: For each $t \in C$ and each $C^{\prime} \in \mathscr{C}^{\prime}$ either $t \in C^{\prime}$ or there is a player $i \in C^{\prime} \backslash C$ such that $t>i$. The existence of coalition $C$ is guaranteed by Proposition 16. One must take $C$ to be the maximal element of the set $\mathscr{C}^{\prime}$ in the lexicographic order induced by the response sequence $>$, as in Definition 5 . We show that each player $t$ of $C$ has a node $h$ where player $t$ 's vote is pivotal.

Take a player $t \in C$. Let $h$ be any node where (a) player $t$ has to react to the proposal $x$, (b) each player $i \in C$ preceding player $t$ in the response order $>$ accepts $x$, and (c) each player $i \in N \backslash C$ preceding player $t$ in the response order $>$ rejects $x$.

Notice that player $t$ accepts the proposal $x$ under the strategy $\sigma_{t}$. We now show that the proposal $x$ is accepted by the coalition $C$ on the path of play induced by the joint strategy strategy $\sigma$ in the subgame $\Gamma(h)$. Indeed, each player $i \in C$ preceding player $t$ in the response order $>\operatorname{accepts} x$ by the definition of the node $h$. And all players $i \in C$ following player $t$ in the response order accept the proposal $x$ along the path of play induced by the joint strategy $\sigma$, because $x \in A_{i}$. 
Consider the strategy $\sigma_{t}^{\prime}$ that coincides with the strategy $\sigma_{t}$ on all nodes but $h$ where it rejects the proposal $x$. We show that the proposal $x$ is socially rejected on the path of play induced by the joint strategy $\left(\sigma_{t}^{\prime}, \sigma_{-t}\right)$ in the subgame $\Gamma(h)$. To see this, let $C^{\prime} \in \mathscr{C}$. Suppose first that $C^{\prime} \in \mathscr{C}^{\prime}$. Player $t$, if a member of coalition $C^{\prime}$, rejects $x$ at the node $h$ by the definition of the strategy $\sigma_{t}^{\prime}$. And if $t$ is not a member of $C^{\prime}$, then there is a player $i \in C^{\prime} \backslash C$ such that $t>i$. Any such player $i$ rejects the proposal $x$ at the node $h$ by condition (c). In either case the proposal $x$ is rejected by at least one of the players of the coalition $C^{\prime}$. Suppose now $C^{\prime}$ is not in $\mathscr{C}^{\prime}$, so there exists a player $i \in C^{\prime}$ such that $x$ is not an element of $A_{i}$. If $i$ follows $t$ in the response order, then $i$ rejects the proposal $x$ along the path of play induced by the strategy $\sigma$. Suppose player $i$ precedes player $t$ in the response order. In this case, note that player $i$ is not an element of the coalition $C$, because $x \in A_{C}$, so player $i$ rejects the proposal $x$ by the choice of the node $h$.

\section{References}

[1] Jeffrey S. Banks and John Duggan: A Bargaining Model of Collective Choice. American Political Science Review, 94: 73-88, 2000.

[2] Ken Binmore, Ariel Rubinstein, and Asher Wolinsky: The Nash Bargaining Solution in Economic Modelling. The RAND Journal of Economics, 17: 176-188, 1986.

[3] Daniel Cardona and Clara Ponsatí: Bargaining One-Dimensional Social Choices. Journal of Economic Theory, doi: 10.1016/j.jet.2006.12.001, 2007.

[4] Seok-Ju Cho and John Duggan: Uniqueness of Stationary Equilibria in a OneDimensional Model of Bargaining. Journal of Economic Theory, 113: 118-130, 2003.

[5] Hülya Eraslan: Uniqueness of Stationary Equilibrium Payoffs in the BaronFerejohn Model. Journal of Economic Theory, 103: 11-30, 2002.

[6] Hülya Eraslan and Antonio Merlo: Majority Rule in a Stochastic Model of Bargaining. Journal of Economic Theory, 103: 31-48, 2002.

[7] Tasos Kalandrakis: Regularity of pure strategy equilibrium points in a class of bargaining games. Economic Theory, 28: 309-329, 2006.

[8] Klaus Kultti and Hannu Vartiainen: Von Neumann-Morgenstern stable sets, discounting, and Nash bargaining. Journal of Economic Theory, doi: 10.1016/j.jet.2006.11.006, 2007.

[9] Antonio Merlo and Charles Wilson: A Stochastic Model of Sequential Bargaining with Complete Information. Econometrica, 63: 371-399, 1995. 
[10] Antonio Merlo and Charles Wilson: Effcient delays in a stochastic model of bargaining. Economic Theory, 11: 39-55, 1998.

[11] ToshiJi Miyakawa: Non-cooperative Foundation of $n$-Person Asymmetric Nash Bargaining Solution. Osaka University of Economics, working paper No. 2006-2.

[12] Arkadi Predtetchinski: One-dimensional bargaining with unanimity rule. Maastricht University, Research Memorandum 07/011. 